MATHEMATICS OF COMPUTATION

Volume 75 , Number 254 , Pages 743-766

S 0025-5718(05)01795-3

Article electronically published on November 3, 2005

\title{
HERMITE AND HERMITE-FEJÉR INTERPOLATION FOR STIELTJES POLYNOMIALS
}

\author{
H. S. JUNG
}

AbStract. Let $w_{\lambda}(x):=\left(1-x^{2}\right)^{\lambda-1 / 2}$ and $P_{n}^{(\lambda)}$ be the ultraspherical polynomials with respect to $w_{\lambda}(x)$. Then we denote by $E_{n+1}^{(\lambda)}$ the Stieltjes polynomials with respect to $w_{\lambda}(x)$ satisfying

$$
\int_{-1}^{1} w_{\lambda}(x) P_{n}^{(\lambda)}(x) E_{n+1}^{(\lambda)}(x) x^{m} d x \begin{cases}=0, & 0 \leq m<n+1 \\ \neq 0, & m=n+1\end{cases}
$$

In this paper, we show uniform convergence of the Hermite-Fejér interpolation polynomials $H_{n+1}[\cdot]$ and $\mathcal{H}_{2 n+1}[\cdot]$ based on the zeros of the Stieltjes polynomials $E_{n+1}^{(\lambda)}$ and the product $E_{n+1}^{(\lambda)} P_{n}^{(\lambda)}$ for $0 \leq \lambda \leq 1$ and $0 \leq \lambda \leq$ $1 / 2$, respectively. To prove these results, we prove that the Lebesgue constants of Hermite-Fejér interpolation operators for the Stieltjes polynomials $E_{n+1}^{(\lambda)}$ and the product $E_{n+1}^{(\lambda)} P_{n}^{(\lambda)}$ are optimal, that is, the Lebesgue constants $\left\|H_{n+1}\right\|_{\infty}(0 \leq \lambda \leq 1)$ and $\left\|\mathcal{H}_{2 n+1}\right\|_{\infty}(0 \leq \lambda \leq 1 / 2)$ have optimal order $O(1)$. In the case of the Hermite-Fejér interpolation polynomials $\mathcal{H}_{2 n+1}[\cdot]$ for $1 / 2<\lambda \leq 1$, we prove weighted uniform convergence. Moreover, we give some convergence theorems of Hermite-Fejér and Hermite interpolation polynomials for $0 \leq \lambda \leq 1$ in weighted $L_{p}$ norms.

\section{INTRODUCTION}

For a function $f:(a, b) \rightarrow \mathbb{R},-\infty \leq a<b \leq \infty$, and a set

$$
\chi_{n}:=\left\{x_{1 n}, x_{2 n}, \ldots, x_{n n}\right\}, n \geq 1,
$$

of pairwise distinct nodes, let $H_{n}\left[\chi_{n} ; f\right]$ and $\hat{H}_{n}\left[\chi_{n} ; f\right]$ denote the Hermite-Fejér and Hermite interpolation polynomials of degree $\leq 2 n-1$ to $f$ with respect to $\chi_{n}$. For the case of Hermite interpolation, we will always assume that $f$ is differentiable so that $\hat{H}_{n}\left[\chi_{n} ; f\right]$ is well defined. In fact, $H_{n}\left[\chi_{n} ; f\right]$ and $\hat{H}_{n}\left[\chi_{n} ; f\right]$ are the unique polynomials of degree $\leq 2 n-1$ satisfying

$$
\left\{\begin{array} { l } 
{ H _ { n } [ \chi _ { n } ; f ] ( x _ { j n } ) = f ( x _ { j n } ) , } \\
{ H _ { n } ^ { \prime } [ \chi _ { n } ; f ] ( x _ { j n } ) = 0 , }
\end{array} \quad \left\{\begin{array}{l}
\hat{H}_{n}\left[\chi_{n} ; f\right]\left(x_{j n}\right)=f\left(x_{j n}\right), \\
\hat{H}_{n}^{\prime}\left[\chi_{n} ; f\right]\left(x_{j n}\right)=f^{\prime}\left(x_{j n}\right)
\end{array}\right.\right.
$$

for $j=1,2, \ldots, n$.

Received by the editor March 9, 2004 and, in revised form, January 12, 2005.

2000 Mathematics Subject Classification. Primary 41A05, 65D05.

Key words and phrases. Generalized Stieltjes polynomial, Hermite-Fejér and Hermite interpolations, convergence, Lebesgue constant.

This work was supported by Korea Research Foundation Grant (KRF-2002-050-C00003). The research on this project started when the author visited Professor Sven Ehrich in GSF-IBB. The author thanks Professor Sven Ehrich, Professor G. Mastroianni, and the referees for many kind suggestions and comments.

(C)2005 American Mathematical Society 743

Reverts to public domain 28 years from publication 
Here, we are interested in Hermite-Fejér and Hermite interpolations with respect to $\chi_{n}$ whose elements are the zeros of a sequence of Stieltjes polynomials. To be precise, we first consider the generalized Stieltjes polynomials $E_{n+1}^{(\lambda)}(x)$. In 1934, Szegő [25] introduced the generalized Stieltjes polynomials $E_{n+1}^{(\lambda)}(x)$ defined by

$$
\int_{-1}^{1} w_{\lambda}(x) P_{n}^{(\lambda)}(x) E_{n+1}^{(\lambda)}(x) x^{k} d x=0, k=0,1, \ldots, n, n \geq 1,
$$

where $w_{\lambda}(x)=\left(1-x^{2}\right)^{\lambda-1 / 2}, \lambda>-1 / 2$, and $P_{n}^{(\lambda)}(x)$ is the ultraspherical (or Gegenbauer) polynomial. The classical Stieltjes polynomials for the Legendre weight $(\lambda=1 / 2)$ were first considered by Stieltjes in 1894, and he made the conjecture that the zeros of $E_{n+1}^{(1 / 2)}(x)$ should all be in $(-1,1)$ and should alternate with those of the $n$-th Legendre polynomial. In [25], Szegö proved, for $0<\lambda \leq 2$, two conjectures of Stieltjes, namely, that the zeros of $E_{n+1}^{(\lambda)}$ are real and inside $(-1,1)$, and that they interlace with the zeros of $P_{n}^{(\lambda)}$.

After Szegö's works, in 1964 Kronrod introduced the so-called Gauss-Kronrod quadrature formulas,

$$
\int_{-1}^{1} w_{\lambda}(x) f(x) d x=\sum_{\nu=1}^{n} A_{\nu, n}^{G K} f\left(x_{\nu, n}^{(\lambda)}\right)+\sum_{\mu=1}^{n+1} B_{\mu, n+1}^{G K} f\left(\xi_{\mu, n+1}^{(\lambda)}\right)+R_{2 n+1}^{G K}(f)
$$

for estimating the error of the Gaussian quadrature formula, and in 1970 Barrucand [1] observed that the zeros of Stieltjes polynomials are precisely the additional nodes $\xi_{1, n+1}^{(\lambda)}, \ldots, \xi_{n+1, n+1}^{(\lambda)}$ of Gauss-Kronrod quadrature formulas. This induced much further study of Stieltjes polynomials and Gauss-Kronrod quadrature formulas (see 3. 5, 6, 8, 17, 21, 9, 12, 13, 22]). These days, Gauss-Kronrod formulas are used extensively in numerical integration software (cf., e.g., [16, 24]). There are the exhaustive surveys of Gautschi 7], Monegato [14, 15, Ehrich [4], Notaris [18, and Peherstorfer [23] for the precise definition of Gauss-Kronrod quadrature formulas and an overview about their connection to Stieltjes polynomials.

For the properties of interpolation operators based at the zeros of $E_{n+1}^{(\lambda)}$ and $P_{n}^{(\lambda)} E_{n+1}^{(\lambda)}$, Ehrich and Mastroianni [5, 6] proved that Lagrange interpolation operators $L_{n+1}$ based on the zeros of $E_{n+1}^{(\lambda)}$ and that extended Lagrange interpolation operators $\mathcal{L}_{2 n+1}$ based on the zeros of $E_{n+1}^{(\lambda)} P_{n}^{(\lambda)}$ have Lebesgue constants $\left\|L_{n+1}\right\|_{\infty}=\sup _{\|f\|_{\infty}}\left\|L_{n+1} f\right\|_{\infty}(0 \leq \lambda \leq 1)$ and $\left\|\mathcal{L}_{2 n+1}\right\|_{\infty}(0 \leq \lambda \leq 1 / 2)$ of optimal order, i.e., $O(\log n)$. So, they showed that the zeros of Stieltjes polynomials improve the Lagrange interpolation process based on the zeros of ultraspherical polynomials, for which Lebesgue constants are well known to be of precise order $O\left(n^{\lambda}\right)$ and $O(\log n)$, according as $0<\lambda$ or $\lambda \leq 0$. See [26, 14.4]. In this paper, we consider the Hermite-Fejér interpolation operator $H_{n+1}$ based on the zeros of $E_{n+1}^{(\lambda)}$ and the extended Hermite-Fejér interpolation operator $\mathcal{H}_{2 n+1}$ based on the zeros of $E_{n+1}^{(\lambda)} P_{n}^{(\lambda)}$, and we show that these interpolation operators have Lebesgue constants $\left\|H_{n+1}\right\|_{\infty}(0 \leq \lambda \leq 1)$ and $\left\|\mathcal{H}_{2 n+1}\right\|_{\infty}(0 \leq \lambda \leq 1 / 2)$ of optimal order, i.e., $O(1)$. See (3.21) and (3.29) in Section 3. From these results, we show uniform convergence of the Hermite-Fejér interpolation polynomials $H_{n+1}[\cdot]$ and $\mathcal{H}_{2 n+1}[\cdot]$ for $0 \leq \lambda \leq 1$ and $0 \leq \lambda \leq 1 / 2$, respectively. In the case of the Hermite-Fejér interpolation polynomials $\mathcal{H}_{2 n+1}[\cdot]$ for $1 / 2<\lambda \leq 1$, we prove weighted uniform 
convergence. Moreover, we prove some convergence theorems of Hermite-Fejér and Hermite interpolation polynomials in weighted $L_{p}$ norms. Let us mention that the Lebesgue constants of the Hermite-Fejér interpolation processes based on the zeros of ultraspherical polynomials are known to be of precise order $O\left(n^{\max \{0,2 \lambda-1\}}\right)$ and that Hermite-Fejér interpolation polynomials are in general divergent at $x=+1$ if $f(x)$ is merely continuous on $[-1,1]$ and $\lambda \geq 1 / 2$. See [26, 14.6] and [27].

\section{MAIN RESULTS}

For the ultraspherical polynomials $P_{n}^{(\lambda)}, \lambda \neq 0$, we use the normalization $P_{n}^{(\lambda)}(1)=\left(\begin{array}{c}n+2 \lambda-1 \\ n\end{array}\right)=O\left(n^{2 \lambda-1}\right)$. We denote the zeros of $P_{n}^{(\lambda)}$ and $E_{n+1}^{(\lambda)}$ by

$$
x_{\nu, n}^{(\lambda)}=\cos \phi_{\nu, n}^{(\lambda)}, \quad \nu=1, \ldots, n \text { and } \xi_{\mu, n+1}^{(\lambda)}=\cos \theta_{\mu, n+1}^{(\lambda)}, \quad \mu=1, \ldots, n+1,
$$

respectively. We denote the zeros of $F_{2 n+1}^{(\lambda)}:=P_{n}^{(\lambda)} E_{n+1}^{(\lambda)}$ by $y_{\nu, 2 n+1}^{(\lambda)}=\cos \psi_{\nu, 2 n+1}^{(\lambda)}$, $\nu=1, \ldots, 2 n+1$. All nodes are ordered by increasing magnitude. We also set

$$
\varphi(x):=\sqrt{1-x^{2}}
$$

and for any two sequences $\left\{b_{n}\right\}_{n}$ and $\left\{c_{n}\right\}_{n}$ of nonzero real numbers (or functions), we write $b_{n} \lesssim c_{n}$ if there exists a constant $C>0$, independent of $n$ (and $x$ ) such that $b_{n} \leq C c_{n}$ for $n$ large enough and write $b_{n} \sim c_{n}$ if $b_{n} \lesssim c_{n}$ and $c_{n} \lesssim b_{n}$. We denote by $\mathcal{P}_{n}$ the space of polynomials of degree at most $n$.

Let $H_{n+1}[;]$ and $\hat{H}_{n+1}[;]$ be the Hermite-Fejér and Hermite interpolation polynomials with respect to the zeros of $E_{n+1}^{(\lambda)}(x)$. Then, the Hermite-Fejér interpolation polynomial $H_{n+1}[f]$ of $f$ admits the representation ([26, pp. 330, 331])

$$
H_{n+1}[f](x)=\sum_{k=1}^{n+1} f\left(\xi_{k, n+1}^{(\lambda)}\right)\left[1-\frac{E_{n+1}^{(\lambda)}{ }^{\prime \prime}\left(\xi_{k, n+1}^{(\lambda)}\right)}{E_{n+1}^{(\lambda)^{\prime}}\left(\xi_{k, n+1}^{(\lambda)}\right)}\left(x-\xi_{k, n+1}^{(\lambda)}\right)\right] l_{k, n+1}^{2}(x) .
$$

The Hermite interpolation polynomial $\hat{H}_{n+1}[f]$ of $f$ on the other hand admits the representation

$$
\hat{H}_{n+1}[f](x)=H_{n+1}[f](x)+\sum_{k=1}^{n+1}\left(x-\xi_{k, n+1}^{(\lambda)}\right) l_{k, n+1}^{2}(x) f^{\prime}\left(\xi_{k, n+1}^{(\lambda)}\right) .
$$

Also let $\mathcal{H}_{2 n+1}[;]$ and $\hat{\mathcal{H}}_{2 n+1}[;]$ be the Hermite-Fejér and Hermite interpolation polynomials with respect to the zeros of $F_{2 n+1}^{(\lambda)}(x)$. Then, similarly, we have

$$
\mathcal{H}_{2 n+1}[f](x)=\sum_{\nu=1}^{2 n+1} f\left(y_{\nu, 2 n+1}^{(\lambda)}\right)\left[1-\frac{F_{2 n+1}^{(\lambda)}{ }^{\prime \prime}\left(y_{\nu, 2 n+1}^{(\lambda)}\right)}{F_{2 n+1}^{(\lambda)}{ }^{\prime}\left(y_{\nu, 2 n+1}^{(\lambda)}\right)}\left(x-y_{\nu, 2 n+1}^{(\lambda)}\right)\right] l_{\nu, 2 n+1}^{2}(x)
$$

and

$$
\hat{\mathcal{H}}_{2 n+1}[f](x)=\mathcal{H}_{2 n+1}[f](x)+\sum_{\nu=1}^{n+1}\left(x-y_{\nu, 2 n+1}^{(\lambda)}\right) l_{\nu, 2 n+1}^{2}(x) f^{\prime}\left(y_{\nu, 2 n+1}^{(\lambda)}\right) .
$$

Here, the fundamental Lagrange interpolation polynomials $l_{k, n+1}(x)$ and $l_{\nu, 2 n+1}(x)$ are given by

$$
l_{k, n+1}(x)=\frac{E_{n+1}^{(\lambda)}(x)}{{E_{n+1}^{(\lambda)}{ }^{\prime}\left(\xi_{k, n+1}^{(\lambda)}\right)\left(x-\xi_{k, n+1}^{(\lambda)}\right)}^{(\lambda)}}, \quad k=1,2, \ldots, n+1
$$


and

$$
l_{\nu, 2 n+1}(x)=\frac{F_{2 n+1}^{(\lambda)}(x)}{F_{2 n+1}^{(\lambda)}{ }^{\prime}\left(y_{\nu, 2 n+1}^{(\lambda)}\right)\left(x-y_{\nu, 2 n+1}^{(\lambda)}\right)}, \quad \nu=1,2, \ldots, 2 n+1 .
$$

In addition, we introduce some basic notations used here and in the following sections. Following usual notation, we write $f \in L_{p}(E), E \subset[-1,1], 0<p<\infty$, if

$$
\|f\|_{L_{p}(E)}=\left(\int_{E}|f(x)|^{p} d x\right)^{1 / p}<\infty,
$$

and we set $L_{p}=L_{p}([-1,1]),\|f\|_{p}=\|f\|_{L_{p}([-1,1])}$. For $p=\infty$, i.e., uniform approximation, we set $\|f\|_{L_{\infty}(E)}=\sup _{x \in E}|f(x)|, E \subset[-1,1]$. In this paper, we need the following notations: For $1 \leq p \leq \infty$,

$$
\omega_{\varphi, p}(f, t):=\sup _{0<h \leq t}\left\|\Delta_{h \varphi} f(x)\right\|_{L_{p}[-1,1]}
$$

and

$$
\mathcal{E}_{n, p}(f):=\inf _{P_{n} \in \mathcal{P}_{n}}\left\|f-P_{n}\right\|_{L_{p}[-1,1]}
$$

where

$$
\Delta_{h \varphi} f(x):=f(x+h \varphi(x) / 2)-f(x-h \varphi(x) / 2) .
$$

In the following subsections, we state our main results: First note that for $\lambda=0$ and $\lambda=1$,

$$
\begin{aligned}
& E_{n+1}^{(0)}(x)=\frac{2 n}{\pi}\left(T_{n+1}(x)-T_{n-1}(x)\right), \\
& E_{n+1}^{(1)}(x)=\frac{2}{\pi} T_{n+1}(x) .
\end{aligned}
$$

In this paper, we let

$$
P_{n}^{(0)}(x):=T_{n}(x) / n \text {. }
$$

\subsection{Uniform convergence of the Hermite-Fejér interpolation polynomi-} als.

Theorem 2.1. Let $\lambda \in[0,1]$ and let $f$ be a continuous function on $[-1,1]$. Then, uniformly for $x \in[-1,1]$,

$$
\lim _{n \rightarrow \infty}\left|H_{n+1}[f](x)-f(x)\right|=0 .
$$

Moreover, we have

$$
\left|H_{n+1}[f](x)-f(x)\right| \lesssim \min \left\{\omega_{\varphi, \infty}\left(f, \frac{\log n}{n}\right), \omega_{\varphi, \infty}\left(f, \frac{1}{n}\right) \log n\right\} .
$$

Theorem 2.2. Let $f$ be a continuous function on $[-1,1]$. If $\lambda \in[0,1 / 2]$, then uniformly for $x \in[-1,1]$,

$$
\lim _{n \rightarrow \infty}\left|\mathcal{H}_{2 n+1}[f](x)-f(x)\right|=0 .
$$

Moreover, we have

$$
\left|\mathcal{H}_{2 n+1}[f](x)-f(x)\right| \lesssim \min \left\{\omega_{\varphi, \infty}\left(f, \frac{\log n}{n}\right), \omega_{\varphi, \infty}\left(f, \frac{1}{n}\right) \log n\right\} .
$$


If $\lambda \in(1 / 2,1]$, then uniformly for $x \in[-1,1]$,

$$
\lim _{n \rightarrow \infty}\left|\left[\mathcal{H}_{2 n+1}[f](x)-f(x)\right] \varphi^{2(2 \lambda-1)}(x)\right|=0 .
$$

Moreover, we have

$$
\begin{aligned}
& \left|\left(\mathcal{H}_{2 n+1}[f](x)-f(x)\right) \varphi^{2(2 \lambda-1)}(x)\right| \\
& \quad \lesssim \min \left\{\omega_{\varphi, \infty}\left(f, \frac{\log n}{n}\right), \omega_{\varphi, \infty}\left(f, \frac{1}{n}\right) \log n\right\} .
\end{aligned}
$$

2.2. Weighted $L_{p}$-convergence of the Hermite-Fejér interpolation polynomials. Let $u$ be a Generalized Jacobi (GJ) weight, defined by

$$
u(x)=\prod_{k=0}^{r}\left|t_{k}-x\right|^{\gamma_{k}}, \quad \gamma_{k}>-1, \quad-1=t_{0}<t_{1}<\cdots<t_{r-1}<t_{r}=1, \quad|x| \leq 1 .
$$

Theorem 2.3. Let $\lambda \in[0,1], 1<p<\infty, u \in L_{p}$, and let $f$ be a continuous function on $[-1,1]$. Then

$$
\left\|\left(H_{n+1}[f](x)-f(x)\right) u\right\|_{p} \lesssim \omega_{\varphi, \infty}\left(f, \frac{1}{n}\right) .
$$

Theorem 2.4. Let $\lambda \in[0,1], 1<p<\infty, u(x)\left(\varphi^{2(1-2 \lambda)}(x)+1\right) \in L_{p}$, and let $f$ be a continuous function on $[-1,1]$. Then

$$
\left\|\left(\mathcal{H}_{2 n+1}[f](x)-f(x)\right) u\right\|_{p} \lesssim \omega_{\varphi, \infty}\left(f, \frac{1}{n}\right) .
$$

2.3. Uniform and $L_{p}$-convergence of the Hermite interpolation polynomials.

Theorem 2.5. Let $\lambda \in[0,1]$ and let $N$ be an integer with $N \geq 1$. If we assume that $f \in C^{N}[-1,1]$ and $0 \leq j \leq N$, then we have

$$
\left\|\left(\hat{H}_{n+1}^{(j)}[f]-f^{(j)}\right) \varphi^{j}\right\|_{\infty} \lesssim \frac{\mathcal{E}_{2 n+1-N, \infty}\left(f^{(N)}\right) \log n}{n^{N-j}} .
$$

Theorem 2.6. Let $\lambda \in[0,1]$ and let $N$ be an integer with $N \geq 1$. Assume that $f \in C^{N}[-1,1]$ and $0 \leq j \leq N$. Then for $\lambda \in[0,1 / 2]$,

$$
\left\|\left(\hat{\mathcal{H}}_{2 n+1}^{(j)}[f]-f^{(j)}\right) \varphi^{j}\right\|_{\infty} \lesssim \frac{\mathcal{E}_{4 n+1-N, \infty}\left(f^{(N)}\right) \log n}{n^{N-j}},
$$

and for $\lambda \in(1 / 2,1]$,

$$
\left\|\left(\hat{\mathcal{H}}_{2 n+1}^{(j)}[f]-f^{(j)}\right) \varphi^{2(2 \lambda-1)+j}\right\|_{\infty} \lesssim \frac{\mathcal{E}_{4 n+1-N, \infty}\left(f^{(N)}\right) \log n}{n^{N-j}} .
$$

Theorem 2.7. Let $\lambda \in[0,1], 1<p<\infty$, and let $N$ be an integer with $N \geq 1$. If we assume that $f \in C^{N}[-1,1]$ and $0 \leq j \leq N$, then we have

$$
\left\|\left(\hat{H}_{n+1}^{(j)}[f]-f^{(j)}\right) \varphi^{j}\right\|_{p} \lesssim \frac{\mathcal{E}_{2 n+1-N, \infty}\left(f^{(N)}\right)}{n^{N-j}} .
$$

Theorem 2.8. Let $\lambda \in[0,1], 1<p<\infty$, and let $N$ be an integer with $N \geq 1$. Assume that $f \in C^{N}[-1,1]$ and $0 \leq j \leq N$. Then for $\lambda \in[0,1 / 2]$,

$$
\left\|\left(\hat{\mathcal{H}}_{2 n+1}^{(j)}[f]-f^{(j)}\right) \varphi^{j}\right\|_{p} \lesssim \frac{\mathcal{E}_{4 n+1-N, \infty}\left(f^{(N)}\right)}{n^{N-j}},
$$


and for $\lambda \in(1 / 2,1]$,

$$
\left\|\left(\hat{\mathcal{H}}_{2 n+1}^{(j)}[f]-f^{(j)}\right) \varphi^{2(2 \lambda-1)+j}\right\|_{p} \lesssim \frac{\mathcal{E}_{4 n+1-N, \infty}\left(f^{(N)}\right)}{n^{N-j}} .
$$

\section{THE PROOFS}

We first state some known results for the Stieltjes polynomials. For $0<\lambda \leq 1$ Ehrich and Mastroianni [5] proved that for $\mu=0,1, \ldots, n+2$ and $\nu=0,1, \ldots, 2 n+$ 2 ,

$$
\left|\theta_{\mu, n+1}^{(\lambda)}-\theta_{\mu+1, n+1}^{(\lambda)}\right| \sim\left|\psi_{\nu, 2 n+1}^{(\lambda)}-\psi_{\nu+1,2 n+1}^{(\lambda)}\right| \sim n^{-1},
$$

where $\psi_{0,2 n+1}^{(\lambda)}:=\theta_{0, n+1}^{(\lambda)}:=\pi$ and $\psi_{2 n+2,2 n+1}^{(\lambda)}:=\theta_{n+2, n+1}^{(\lambda)}:=0$. It implies that for $\mu=0,1, \ldots, n+2$ and $\nu=0,1, \ldots, 2 n+2$,

$$
\left\{\begin{array} { l } 
{ \xi _ { \mu + 1 , n + 1 } ^ { ( \lambda ) } - \xi _ { \mu , n + 1 } ^ { ( \lambda ) } \sim \frac { 1 } { n } \varphi ( \xi _ { \mu , n + 1 } ^ { ( \lambda ) } ) , } \\
{ y _ { \nu + 1 , 2 n + 1 } ^ { ( \lambda ) } - y _ { \nu , 2 n + 1 } ^ { ( \lambda ) } \sim \frac { 1 } { n } \varphi ( y _ { \nu , 2 n + 1 } ^ { ( \lambda ) } ) }
\end{array} \quad \text { and } \quad \left\{\begin{array}{l}
\varphi\left(\xi_{\mu+1, n+1}^{(\lambda)}\right) \sim \varphi\left(\xi_{\mu, n+1}^{(\lambda)}\right), \\
\varphi\left(y_{\nu+1,2 n+1}^{(\lambda)}\right) \sim \varphi\left(y_{\nu, 2 n+1}^{(\lambda)}\right),
\end{array}\right.\right.
$$

where $y_{0,2 n+1}^{(\lambda)}:=\xi_{0, n+1}^{(\lambda)}:=-1$ and $y_{2 n+2,2 n+1}^{(\lambda)}:=\xi_{n+2, n+1}^{(\lambda)}:=1$.

In the following, we list some propositions about estimates of $E_{n+1}^{(\lambda)}(x)$ and $F_{2 n+1}^{(\lambda)}(x)$ from [5, 9].

Proposition $3.1([5])$. Let $0<\lambda \leq 1$. Then

$$
\left|E_{n+1}^{(\lambda)}(x)\right| \lesssim n^{1-\lambda} \varphi^{1-\lambda}(x)+1, \quad-1 \leq x \leq 1,
$$

and $E_{n+1}^{(\lambda)}(1) \gtrsim 1$. Moreover, we have

$$
\left|F_{2 n+1}^{(\lambda)}(x)\right| \lesssim \varphi^{1-2 \lambda}(x), \quad \xi_{1, n+1}^{(\lambda)} \leq x \leq \xi_{n+1, n+1}^{(\lambda)},
$$

and $\left|F_{2 n+1}^{(\lambda)}(1)\right| \sim n^{2 \lambda-1}$. For $\lambda=0$, we know that

$$
\left|E_{n+1}^{(0)}(x)\right| \lesssim n \varphi(x), \quad-1 \leq x \leq 1
$$

and

$$
\left|F_{2 n+1}^{(0)}(x)\right| \lesssim \varphi(x), \quad-1 \leq x \leq 1 .
$$

Proof. For $0<\lambda<1$, see [5]. For $\lambda=0,1$, the assertion can be proved easily by (2.5), (2.6) and (2.7).

Proposition $3.2([9])$. Let $0<\lambda \leq 1$. Then for $x \in\left[\xi_{1, n+1}^{(\lambda)}, \xi_{n+1, n+1}^{(\lambda)}\right]$,

$$
\left|E_{n+1}^{(\lambda)}{ }^{\prime}(x)\right| \lesssim n^{2-\lambda} \varphi^{-\lambda}(x)
$$

Moreover, we have for $x \in\left[-1, \xi_{1, n+1}^{(\lambda)}\right] \cup\left[\xi_{n+1, n+1}^{(\lambda)}, 1\right]$,

$$
\left|E_{n+1}^{(\lambda)}{ }^{\prime}(x)\right| \sim n^{2} .
$$

In the case of $\lambda=0$, we know that for $|x| \leq 1$,

$$
\left|E_{n+1}^{(0)}{ }^{\prime}(x)\right| \lesssim n^{2},
$$


and for $\cos ^{-1} \frac{\pi}{2 n} \leq|x| \leq 1$,

$$
\left|E_{n+1}^{(0)}{ }^{\prime}(x)\right| \sim n^{2} .
$$

Proof. For $0<\lambda<1$, see [9]. For $\lambda=0,1$, the estimates follow by (2.5).

Proposition $3.3([9])$. Let $0<\lambda \leq 1$. Then for $x \in\left[\xi_{1, n+1}^{(\lambda)}, \xi_{n+1, n+1}^{(\lambda)}\right]$,

$$
\left|F_{2 n+1}^{(\lambda)}{ }^{\prime}(x)\right| \lesssim n \varphi^{-2 \lambda}(x) .
$$

Moreover, we have for $x \in\left[-1, \xi_{1, n+1}^{(\lambda)}\right] \cup\left[\xi_{n+1, n+1}^{(\lambda)}, 1\right]$,

$$
\left|F_{2 n+1}^{(\lambda)}{ }^{\prime}(x)\right| \sim n^{1+2 \lambda} .
$$

In the case of $\lambda=0$, we know that for $|x| \leq 1$,

$$
\left|F_{2 n+1}^{(0)}{ }^{\prime}(x)\right| \lesssim n,
$$

and for $\cos ^{-1} \frac{\pi}{4 n} \leq|x| \leq 1$,

$$
\left|F_{2 n+1}^{(0)}{ }^{\prime}(x)\right| \sim n .
$$

Proof. For $0<\lambda<1$, see [9]. For $\lambda=0,1$, the assertion can be proved easily by (2.5), (2.6) and (2.7).

Proposition $3.4\left([]_{0}\right)$. Let $0 \leq \lambda \leq 1$. Then for $\mu=1,2, \ldots, n+1$,

$$
\left|E_{n+1}^{(\lambda)}{ }^{\prime}\left(\xi_{\mu, n+1}^{(\lambda)}\right)\right| \sim n^{2-\lambda} \varphi^{-\lambda}\left(\xi_{\mu, n+1}^{(\lambda)}\right),
$$

and for $\nu=1,2, \ldots, 2 n+1$,

$$
\left|F_{2 n+1}^{(\lambda)}{ }^{\prime}\left(y_{\nu, 2 n+1}^{(\lambda)}\right)\right| \sim n \varphi^{-2 \lambda}\left(y_{\nu, 2 n+1}^{(\lambda)}\right) .
$$

Proof. For $0<\lambda<1$, see [5]. For $\lambda=0,1$, the assertion can be proved easily by (2.5), (2.6), and (2.7).

Proposition $3.5(9])$. Let $0<\lambda \leq 1$. Then for $\mu=1,2, \ldots, n+1$,

$$
\left|E_{n+1}^{(\lambda)}{ }^{\prime \prime}\left(\xi_{\mu, n+1}^{(\lambda)}\right)\right| \lesssim n^{2} \varphi^{-2}\left(\xi_{\mu, n+1}^{(\lambda)}\right),
$$

and for $\nu=1,2, \ldots, 2 n+1$,

$$
\left|F_{2 n+1}^{(\lambda)}{ }^{\prime \prime}\left(y_{\nu, 2 n+1}^{(\lambda)}\right)\right| \lesssim n^{1+\lambda} \varphi^{-2-\lambda}\left(y_{\nu, 2 n+1}^{(\lambda)}\right) .
$$

In the case of $\lambda=0$, we know that for $\mu=2, \ldots, n$ and $\nu=2, \ldots, 2 n$, (3.10) and (3.11) are satisfied, and for $\mu=1, n+1$ and $\nu=1,2 n+1$,

$$
\left|E_{n+1}^{(0)}{ }^{\prime \prime}\left(\xi_{\mu, n+1}^{(0)}\right)\right| \sim n^{4} \quad \text { and } \quad\left|F_{2 n+1}^{(0)}{ }^{\prime \prime}\left(y_{\nu, 2 n+1}^{(0)}\right)\right| \sim n^{3} .
$$

Proof. For $0<\lambda<1$, see [9]. For $\lambda=0,1$, the assertion can be proved easily by (2.5), (2.6), and (2.7).

For convenience, we let

$$
H_{1, n+1}[f]:=\sum_{k=1}^{n+1} f\left(\xi_{k, n+1}^{(\lambda)}\right) l_{k, n+1}^{2}(x)
$$


and

$$
H_{2, n+1}[f]:=\sum_{k=1}^{n+1} f\left(\xi_{k, n+1}^{(\lambda)}\right) \frac{E_{n+1}^{(\lambda)}{ }^{\prime \prime}\left(\xi_{k, n+1}^{(\lambda)}\right)}{E_{n+1}^{(\lambda)}{ }^{\prime}\left(\xi_{k, n+1}^{(\lambda)}\right)}\left(x-\xi_{k, n+1}^{(\lambda)}\right) l_{k, n+1}^{2}(x) .
$$

Then by (2.1)

$$
H_{n+1}[f]=H_{1, n+1}[f]-H_{2, n+1}[f] .
$$

Similarly, we split $\mathcal{H}_{2 n+1}[f]$ into two terms. Then by (2.3)

$$
\mathcal{H}_{2 n+1}[f]:=\mathcal{H}_{1,2 n+1}[f]-\mathcal{H}_{2,2 n+1}[f] .
$$

3.1. Convergence of the Hermite-Fejér interpolation polynomials for $E_{n+1}^{(\lambda)}(x)$. In this subsection we prove Theorems 2.1 and 2.3.

Lemma 3.6. Let $\lambda \in[0,1]$. Then we have for large $n>0$,

$$
\left|H_{2, n+1}[f](x)\right| \lesssim\|f\|_{\infty} .
$$

Proof. First let $0<\lambda \leq 1$. By [19, (26)] it is sufficient to prove (3.15) for $0 \leq$ $x \leq 1-\frac{a}{n^{2}}$, where $a>0$ is a fixed, sufficiently small constant. In the following let $\|f\|_{\infty}=1$ and let $x \in\left[\xi_{k-1, n+1}^{(\lambda)}, \xi_{k+1, n+1}^{(\lambda)}\right] \cap\left[0,1-\frac{a}{n^{2}}\right]\left(\xi_{0, n+1}^{(\lambda)}:=-1, \xi_{n+2, n+1}^{(\lambda)}:=1\right)$ for some $1 \leq k \leq n+1$. Then,

$$
\begin{aligned}
\left|H_{2, n+1}[f](x)\right| & \lesssim \sum_{j=1}^{n+1}\left|\frac{E_{n+1}^{(\lambda)}{ }^{\prime \prime}\left(\xi_{j, n+1}^{(\lambda)}\right)}{E_{n+1}^{(\lambda)}{ }^{\prime}\left(\xi_{j, n+1}^{(\lambda)}\right)}\right|\left|x-\xi_{j, n+1}^{(\lambda)}\right| l_{j, n+1}^{2}(x) \\
& =\sum_{j \in[k-2, k+2]}+\sum_{j \notin[k-2, k+2]}=: J_{1}+J_{2} .
\end{aligned}
$$

For $j \in[k-2, k+2] \cap[1, n+1]$, there exists some $r_{j, n+1}^{(\lambda)}$ between $x$ and $\xi_{j, n+1}^{(\lambda)}$ such that we have by the mean value property, (3.1), (3.6), and (3.8),

$$
\begin{aligned}
\left|l_{j, n+1}(x)\right| & =\left|\frac{E_{n+1}^{(\lambda)}(x)}{\left(x-\xi_{j, n+1}^{(\lambda)}\right) E_{n+1}^{(\lambda)}{ }^{\prime}\left(\xi_{j, n+1}^{(\lambda)}\right)}\right| \\
& =\left|\frac{E_{n+1}^{(\lambda)}{ }^{\prime}\left(r_{j, n+1}^{(\lambda)}\right)}{E_{n+1}^{(\lambda)}{ }^{\prime}\left(\xi_{j, n+1}^{(\lambda)}\right)}\right| \lesssim \frac{n^{2-\lambda} \varphi^{-\lambda}\left(r_{j, n+1}^{(\lambda)}\right)}{n^{2-\lambda} \varphi^{-\lambda}\left(\xi_{j, n+1}^{(\lambda)}\right)} \lesssim 1 .
\end{aligned}
$$

Then, we obtain by (3.1), (3.8), (3.10), and (3.16),

$$
\begin{aligned}
J_{1} & =\sum_{j \in[k-2, k+2]}\left|\frac{E_{n+1}^{(\lambda)}{ }^{\prime \prime}\left(\xi_{j, n+1}^{(\lambda)}\right)}{E_{n+1}^{(\lambda)}{ }^{\prime}\left(\xi_{j, n+1}^{(\lambda)}\right)}\right|\left|x-\xi_{j, n+1}^{(\lambda)}\right| l_{j, n+1}^{2}(x) \\
& \lesssim \sum_{j \in[k-2, k+2]}\left|\frac{E_{n+1}^{(\lambda)}{ }^{\prime \prime}\left(\xi_{j, n+1}^{(\lambda)}\right)}{E_{n+1}^{(\lambda)}{ }^{\prime}\left(\xi_{j, n+1}^{(\lambda)}\right)}\right|\left|x-\xi_{j, n+1}^{(\lambda)}\right| \\
& \lesssim \frac{n^{2} \varphi^{-2}(x)}{n^{2-\lambda} \varphi^{-\lambda}(x)} \frac{1}{n} \varphi(x) \sim n^{\lambda-1} \varphi^{\lambda-1}(x) \lesssim 1 .
\end{aligned}
$$


Next, we estimate $J_{2}$. Assume $0 \leq x \leq \xi_{n-1, n+1}^{(\lambda)}$. Then for some $C>0$ with $1-\frac{C}{n^{2}}>\frac{\xi_{n+1, n+1}^{(\lambda)}+1}{2}$, we have by (3.1), (3.2), (3.8), and (3.10),

$$
\begin{aligned}
J_{2} & =\left|E_{n+1}^{(\lambda)}(x)\right|^{2} \sum_{j \notin[k-2, k+2]} \frac{\left|E_{n+1}^{(\lambda)}{ }^{\prime \prime}\left(\xi_{j, n+1}^{(\lambda)}\right)\right|}{\left|E_{n+1}^{(\lambda)}{ }^{\prime}\left(\xi_{j, n+1}^{(\lambda)}\right)\right|^{3}\left|x-\xi_{j, n+1}^{(\lambda)}\right|} \\
& \lesssim n^{2(1-\lambda)} \varphi^{2(1-\lambda)}(x) \sum_{j \notin[k-2, k+2]} \frac{\varphi^{3 \lambda-2}\left(\xi_{j, n+1}^{(\lambda)}\right)}{n^{4-3 \lambda}} \frac{1}{\left|x-\xi_{j, n+1}^{(\lambda)}\right|} \\
& \lesssim \frac{\varphi^{2(1-\lambda)}(x)}{n^{1-\lambda}} \sum_{j \notin[k-2, k+2]} \frac{\varphi^{3 \lambda-2}\left(\xi_{j, n+1}^{(\lambda)}\right)}{n} \frac{1}{\left|x-\xi_{j, n+1}^{(\lambda)}\right|} \\
& \lesssim \frac{\varphi^{2(1-\lambda)}(x)}{n^{1-\lambda}} \int_{\left[-1+\frac{C}{n^{2}}, 1-\frac{C}{n^{2}}\right] \backslash\left[\xi_{k-2, n+1}^{(\lambda)}, \xi_{k+2, n+1}^{(\lambda)}\right]} \frac{\varphi^{3(\lambda-1)}(t)}{|x-t|} d t .
\end{aligned}
$$

Then for the integral

$$
\int \frac{\varphi^{3(\lambda-1)}(t)}{|x-t|} d t \lesssim \int_{0}^{x_{*}}+\int_{x^{*}}^{\frac{1+x^{*}}{2}}+\int_{\frac{1+x^{*}}{2}}^{1-\frac{C}{n^{2}}} \frac{\varphi^{3(\lambda-1)}(t)}{|x-t|} d t,
$$

where $x_{*}:=\xi_{k-2, n+1}^{(\lambda)}$ and $x^{*}:=\xi_{k+2, n+1}^{(\lambda)}$. Here, since $\varphi^{\lambda-1}(x)$ is increasing on $(0,1), \varphi\left(x_{*}\right) \sim \varphi(x)$ by (3.1), and $1-\frac{x_{*}}{x} \gtrsim \frac{1}{n^{2}}$, we have

$$
\begin{aligned}
\left|\int_{0}^{x_{*}} \frac{\varphi^{3(\lambda-1)}(t)}{x-t} d t\right| & \lesssim \varphi^{2(\lambda-1)}\left(x_{*}\right)\left|\int_{0}^{x_{*}} \frac{\varphi^{\lambda-1}(t)}{x-t} d t\right| \\
& \lesssim \varphi^{2(\lambda-1)}(x)\left|\int_{0}^{\frac{x_{*}}{x}} \frac{\varphi^{\lambda-1}(x s)}{1-s} d s\right| \\
& \lesssim \varphi^{2(\lambda-1)}(x) \int_{0}^{\left|\frac{x_{*}}{x}\right|}(1-s)^{\frac{\lambda-1}{2}-1} d s \\
& \lesssim \varphi^{2(\lambda-1)}(x) n^{1-\lambda} .
\end{aligned}
$$

Since

$$
\varphi\left(\frac{1+x^{*}}{2}\right) \sim \sqrt{1-\frac{1+x^{*}}{2}} \sim \varphi\left(x^{*}\right) \sim \varphi(x)
$$

we have

$$
\begin{aligned}
\int_{x^{*}}^{\frac{1+x^{*}}{2}} \frac{\varphi^{3(\lambda-1)}(t)}{|x-t|} d t & \lesssim \varphi^{2(\lambda-1)}\left(\frac{1+x^{*}}{2}\right) \int_{x^{*}}^{\frac{1+x^{*}}{2}} \frac{\varphi^{\lambda-1}(t)}{t-x} d t \\
& \lesssim \varphi^{2(\lambda-1)}(x) \int_{x^{*}}^{\frac{1+x^{*}}{2}}(t-x)^{\frac{1-\lambda}{2}-1}+(1-t)^{\frac{1-\lambda}{2}-1} d t \\
& \lesssim \varphi^{2(\lambda-1)}(x) n^{1-\lambda}
\end{aligned}
$$


and since $|x-t| \gtrsim|1-t|$ for $\frac{1+x^{*}}{2}<t<1-\frac{C}{n^{2}}$,

$$
\begin{aligned}
\int_{\frac{1+x^{*}}{2}}^{1-\frac{C}{n^{2}}} \frac{\varphi^{3(\lambda-1)}(t)}{|x-t|} d t & \lesssim \frac{1}{\left|\frac{1+x^{*}}{2}-x\right|^{1-\lambda}} \int_{\frac{1+x^{*}}{2}}^{1-\frac{C}{n^{2}}} \frac{\varphi^{3(\lambda-1)}(t)}{|t-x|^{\lambda}} d t \\
& \lesssim \varphi^{2(\lambda-1)}(x) \int_{\frac{1+x^{*}}{2}}^{1-\frac{C}{n^{2}}} \frac{1}{|t-x|^{\lambda}(1-t)^{\frac{3}{2}-\frac{3 \lambda}{2}}} d t \\
& \lesssim \varphi^{2(\lambda-1)}(x) \int_{\frac{1+x^{*}}{2}}^{1-\frac{C}{n^{2}}} \frac{1}{(1-t)^{\frac{3}{2}-\frac{\lambda}{2}}} d t \\
& \lesssim \varphi^{2(\lambda-1)}(x) n^{1-\lambda} .
\end{aligned}
$$

Thus, we have $J_{2} \lesssim 1$ for $0 \leq x \leq \xi_{n-1, n+1}^{(\lambda)}$. For $\xi_{n-1, n+1}^{(\lambda)}<x \leq 1$ we obtain in the same way as in the case $0 \leq x \leq \xi_{n-1, n+1}^{(\lambda)}$,

$$
J_{2} \lesssim \frac{\varphi^{2(1-\lambda)}(x)}{n^{1-\lambda}} \int_{0}^{x_{*}} \frac{\varphi^{3(\lambda-1)}(t)}{|x-t|} d t \lesssim 1 .
$$

So, we have $J_{2} \lesssim 1$. Therefore, (3.15) is proved for $0<\lambda \leq 1$. Now, we consider the case of $\lambda=0$. Then

$$
\begin{aligned}
\left|H_{2, n+1}[f](x)\right| & \lesssim \sum_{j=2}^{n}+\sum_{j=1, n+1}\left|\frac{E_{n+1}^{(0)}{ }^{\prime \prime}\left(\xi_{j, n+1}^{(0)}\right)}{E_{n+1}^{(0)}{ }^{\prime}\left(\xi_{j, n+1}^{(0)}\right)}\right|\left|x-\xi_{j, n+1}^{(0)}\right| l_{j, n+1}^{2}(x) \\
= & K_{1}+K_{2} .
\end{aligned}
$$

Since by (3.4), (3.8), and (3.12)

$$
\left|\frac{E_{n+1}^{(0)}{ }^{\prime \prime}\left(\xi_{j, n+1}^{(0)}\right)}{{E_{n+1}^{(0)}{ }^{\prime}\left(\xi_{j, n+1}^{(0)}\right)}^{(0)}}\right|\left|x-\xi_{j, n+1}^{(0)}\right| l_{j, n+1}^{2}(x) \lesssim 1 \quad(\text { when } j=1, n+1),
$$

we have $K_{2} \lesssim 1$, and in the same way as in the case $0<\lambda \leq 1$, we have $K_{1} \lesssim 1$. Therefore, (3.15) is proved for $0 \leq \lambda \leq 1$.

Lemma 3.7. Let $\lambda \in(0,1]$. Then we have

$$
\sum_{j=1}^{n+1}\left|l_{j, n+1}(x)\right| \varphi^{1-\lambda}(x) \varphi^{\lambda-1}\left(\xi_{j, n+1}^{(\lambda)}\right) \lesssim \log n .
$$

In the case of $\lambda=0$, we have

$$
\sum_{j=2}^{n}\left|l_{j, n+1}(x)\right| \varphi(x) \varphi^{-1}\left(\xi_{j, n+1}^{(0)}\right) \lesssim \log n .
$$

Proof. First, consider the case of $0<\lambda \leq 1$. Assume that $0 \leq x \leq 1-\frac{a}{n^{2}}$, where $a>$ 0 is a fixed, sufficiently small constant, and let $x \in\left[\xi_{k-1, n+1}^{(\lambda)}, \xi_{k+1, n+1}^{(\lambda)}\right] \cap\left[0,1-\frac{a}{n^{2}}\right]$ $\left(\xi_{0, n+1}^{(\lambda)}:=-1, \xi_{n+2, n+1}^{(\lambda)}:=1\right)$ for some $1 \leq k \leq n+1$. We first split the left equation of (3.17) into $J_{1}$ and $J_{2}$ :

$$
\sum_{j \in[k-2, k+2]}+\sum_{j \notin[k-2, k+2]}\left|l_{j, n+1}(x)\right| \varphi^{1-\lambda}(x) \varphi^{\lambda-1}\left(\xi_{j, n+1}^{(\lambda)}\right)=: J_{1}+J_{2} .
$$


Then by (3.1) and (3.16),

$$
J_{1} \lesssim \sum_{j \in[k-2, k+2] \cap[1, n+1]} \varphi^{1-\lambda}(x) \varphi^{\lambda-1}\left(\xi_{j, n+1}^{(\lambda)}\right) \lesssim 1 .
$$

If we assume that $0 \leq x \leq \xi_{n-1, n+1}^{(\lambda)}$, then by (3.2) and (3.8),

$$
\begin{aligned}
J_{2} & \lesssim \sum_{j \notin[k-2, k+2]} \frac{\left|E_{n+1}^{(\lambda)}(x)\right| \varphi^{1-\lambda}(x) \varphi^{\lambda-1}\left(\xi_{j, n+1}^{(\lambda)}\right)}{\left|E_{n+1}^{(\lambda)}{ }^{\prime}\left(\xi_{j, n+1}^{(\lambda)}\right)\right|}\left|\frac{1}{x-\xi_{j, n+1}^{(\lambda)}}\right| \\
& \lesssim \varphi^{2(1-\lambda)}(x) \sum \frac{\varphi^{2 \lambda-1}\left(\xi_{j, n+1}^{(\lambda)}\right)}{n\left|x-\xi_{j, n+1}^{(\lambda)}\right|} \\
& \lesssim \varphi^{2(1-\lambda)}(x) \int_{[-1,1] \backslash\left[\xi_{k-2, n+1}^{(\lambda)}, \xi_{k+2, n+1}^{(\lambda)}\right]} \frac{\left(1-t^{2}\right)^{\lambda-1}}{|x-t|} d t .
\end{aligned}
$$

To estimate the last integral we also split it into three terms:

$$
\int \frac{\left(1-t^{2}\right)^{\lambda-1}}{|x-t|} d t \lesssim \int_{0}^{x_{*}}+\int_{x^{*}}^{\frac{1+x^{*}}{2}}+\int_{\frac{1+x^{*}}{2}}^{1-\frac{C}{n^{2}}} \frac{\varphi^{2(\lambda-1)}(t)}{|x-t|} d t=: I_{1}+I_{2}+I_{3}
$$

where $x_{*}:=\xi_{k-2, n+1}^{(\lambda)}$ and $x^{*}:=\xi_{k+2, n+1}^{(\lambda)}$. Then, since $\varphi^{2(\lambda-1)}(x)$ is increasing on $(0,1)$, we have

$$
\begin{gathered}
I_{1} \lesssim \varphi^{2(\lambda-1)}\left(x_{*}\right) \int_{0}^{x_{*}} \frac{1}{|x-t|} d t \lesssim \varphi^{2(\lambda-1)}(x) \log n, \\
I_{2} \lesssim \varphi^{2(\lambda-1)}\left(\frac{1+x^{*}}{2}\right) \int_{x^{*}}^{\frac{1+x^{*}}{2}} \frac{1}{|x-t|} d t \lesssim \varphi^{2(\lambda-1)}(x) \log n,
\end{gathered}
$$

and

$$
\begin{aligned}
I_{3} & \lesssim \frac{1}{\left|\frac{1+x^{*}}{2}-x\right|} \int_{\frac{1+x^{*}}{2}}^{1-\frac{C}{n^{2}}} \varphi^{2(\lambda-1)}(t) d t \\
& \lesssim \varphi^{-2}(x)\left(\varphi^{2 \lambda}\left(1-\frac{C}{n^{2}}\right)+\varphi^{2 \lambda}\left(\frac{1+x^{*}}{2}\right)\right) \lesssim \varphi^{2(\lambda-1)}(x) .
\end{aligned}
$$

If $\xi_{n-1, n+1}^{(\lambda)}<x \leq 1$, then we obtain in the same way as in the case $0 \leq x \leq \xi_{n-1, n+1}^{(\lambda)}$,

$$
J_{2} \lesssim \varphi^{2(1-\lambda)}(x) \int_{0}^{x_{*}} \frac{\varphi^{2(\lambda-1)}(t)}{|x-t|} d t \lesssim \log n .
$$

Thus, we have $J_{2} \lesssim \log n$ so that (3.17) is proved for $0<\lambda \leq 1$. For $\lambda=0$, the proof is similar to the case of $0<\lambda \leq 1$ by (3.4) and (3.8). Therefore, (3.18) is also proved.

Lemma 3.8. Let $\lambda \in[0,1]$. For any polynomial $R \in \mathcal{P}_{2 n+1}$,

$$
\left|R(x)-H_{n+1}[R](x)\right| \lesssim\left\|R^{\prime} \varphi\right\|_{\infty} \frac{\log n}{n} .
$$


Proof. Let $0<\lambda \leq 1$. For any polynomial $R \in \mathcal{P}_{2 n+1}$, we have by (2.2), (3.2), (3.8) and (3.17),

$$
\begin{aligned}
\mid R(x) & -H_{n+1}[R](x) \mid \\
= & \left|\hat{H}_{n+1}[R](x)-H_{n+1}[R](x)\right| \\
= & \left|\sum_{j=1}^{n+1}\left(x-\xi_{j, n+1}^{(\lambda)}\right) l_{j, n+1}^{2}(x) R^{\prime}\left(\xi_{j, n+1}^{(\lambda)}\right)\right| \\
= & \left|\sum_{j=1}^{n+1} \frac{E_{n+1}^{(\lambda)}(x)}{E_{n+1}^{(\lambda)}{ }^{\prime}\left(\xi_{j, n+1}^{(\lambda)}\right)} l_{j, n+1}(x) R^{\prime}\left(\xi_{j, n+1}^{(\lambda)}\right)\right| \\
& \lesssim \sum_{j=1}^{n+1} \frac{\varphi^{1-\lambda}(x) \varphi^{\lambda}\left(\xi_{j, n+1}^{(\lambda)}\right)}{n}\left|l_{j, n+1}(x)\right|\left|R^{\prime}\left(\xi_{j, n+1}^{(\lambda)}\right)\right| \\
& \lesssim \frac{\left\|R^{\prime} \varphi\right\|_{\infty}}{n} \sum_{j=1}^{n+1} \frac{\varphi^{1-\lambda}(x) \varphi^{\lambda-1}\left(\xi_{j, n+1}^{(\lambda)}\right)}{n}\left|l_{j, n+1}(x)\right| \\
& \lesssim \frac{\left\|R^{\prime} \varphi\right\|_{\infty}}{n} \log n .
\end{aligned}
$$

Now, we consider the case of $\lambda=0$. Then

$$
\begin{aligned}
\left|R(x)-H_{n+1}[R](x)\right| & \leq \sum_{j=1}^{n+1}\left|\frac{E_{n+1}^{(0)}(x)}{E_{n+1}^{(0)}{ }^{\prime}\left(\xi_{j, n+1}^{(0)}\right)}\right|\left|l_{j, n+1}(x)\right|\left|R^{\prime}\left(\xi_{j, n+1}^{(0)}\right)\right| \\
& =\sum_{j=2}^{n}+\sum_{j=1, n+1}\left|\frac{E_{n+1}^{(0)}(x)}{E_{n+1}^{(0)}{ }^{\prime}\left(\xi_{j, n+1}^{(0)}\right)}\right|\left|l_{j, n+1}(x)\right|\left|R^{\prime}\left(\xi_{j, n+1}^{(0)}\right)\right| \\
& =: K_{1}+K_{2} .
\end{aligned}
$$

Since by (3.4) and (3.8)

$$
K_{2} \lesssim \frac{\left|R^{\prime}(1)\right|+\left|R^{\prime}(-1)\right|}{n^{2}}
$$

and by [19, (26)]

$$
\frac{\left|R^{\prime}(1)\right|+\left|R^{\prime}(-1)\right|}{n} \lesssim \frac{\left\|R^{\prime}\right\|_{\infty}}{n} \lesssim\left\|R^{\prime} \varphi\right\|_{\infty},
$$

we have

$$
K_{2} \lesssim \frac{\left\|R^{\prime} \varphi\right\|_{\infty}}{n} .
$$

For $K_{1}$ we obtain in the same way as in the case $0<\lambda \leq 1$,

$$
K_{1} \lesssim \frac{\left\|R^{\prime} \varphi\right\|_{\infty}}{n} \log n .
$$

Therefore, we have (3.20) for $0 \leq \lambda \leq 1$. 
Proof of Theorem 2.1. Since $H_{n+1}[1](x)=1$, we have by (3.13) and (3.15)

$$
\begin{aligned}
\left|H_{1, n+1}[f](x)\right| & \lesssim\|f\|_{\infty} \sum_{k=1}^{n+1} l_{k, n+1}^{2}(x)=\|f\|_{\infty} H_{1, n+1}[1](x) \\
& =\|f\|_{\infty}\left(H_{n+1}[1](x)+H_{2, n+1}[1](x)\right) \\
& \lesssim\|f\|_{\infty} .
\end{aligned}
$$

Therefore, we have

$$
\left.\left|H_{n+1}[f](x)\right| \lesssim\|f\|_{\infty} \quad \text { (i.e., }\left\|H_{n+1}\right\|_{\infty}=O(1)\right) .
$$

Since $f$ is continuous on $[-1,1]$, for given $\epsilon>0$, there exists a polynomial $R$ such that for $x \in[-1,1]$,

$$
|f(x)-R(x)|<\epsilon .
$$

Then we have from (3.20) and (3.21)

$$
\begin{aligned}
& \lim _{n \rightarrow \infty}\left|H_{n+1}[f](x)-f(x)\right| \\
\leq & |f(x)-R(x)|+\lim _{n \rightarrow \infty}\left|H_{n+1}[f-R](x)\right|+\lim _{n \rightarrow \infty}\left|H_{n+1}[R](x)-R(x)\right| \\
\lesssim & \|f-R\|_{\infty}+\lim _{n \rightarrow \infty}\left\|R^{\prime} \varphi\right\|_{\infty} \frac{\log n}{n} \lesssim \epsilon .
\end{aligned}
$$

Thus, (2.8) is proved, since $\epsilon>0$ is arbitrary. Moreover, since for any polynomial $R \in \mathcal{P}_{\left[\frac{n}{\log n}\right]}$ by the above estimation,

$$
\left|H_{n+1}[f](x)-f(x)\right| \lesssim\|f-R\|_{\infty}+\left\|R^{\prime} \varphi\right\|_{\infty} /\left[\frac{n}{\log n}\right],
$$

we have from [2, Theorem 7.3.1],

$$
\left|H_{n+1}[f](x)-f(x)\right| \lesssim \omega_{\varphi, \infty}\left(f, \frac{\log n}{n}\right) .
$$

Also, since for any polynomial $R \in \mathcal{P}_{2 n+1}$

$$
\left|H_{n+1}[f](x)-f(x)\right| \lesssim\|f-R\|_{\infty}+\left\|R^{\prime} \varphi\right\|_{\infty} \frac{\log n}{n},
$$

we have

$$
\left|H_{n+1}[f](x)-f(x)\right| \lesssim \omega_{\varphi, \infty}\left(f, \frac{1}{n}\right) \log n .
$$

Therefore, (2.9) is proved.

In the following, we use some properties of the Hilbert transform $H(f)$, defined by

$$
H[f, t]:=\lim _{\epsilon \rightarrow 0} \int_{|x-t| \geq \epsilon} \frac{f(x)}{x-t} d x, \quad f \in L_{1} .
$$

We recall that if $G \in L_{\infty}$ and $F \log ^{+} F \in L_{1}$, where $F$ and $G$ have compact support $K$, then we have (cf. [6, 20])

$$
\int_{K} G H[F]=-\int_{K} F H[G] .
$$


Lemma 3.9. Let $\lambda \in[0,1], 1<p<\infty$, and let $u \in L_{p}$. Then for any polynomial $R \in \mathcal{P}_{2 n+1}$

$$
\left\|\left(R-H_{n+1}[R]\right) u\right\|_{L_{p}[-1,1]} \lesssim \frac{\left\|R^{\prime} \varphi\right\|_{L_{\infty}}}{n} .
$$

Proof. We follow the proof of Theorem 3.2 in [6]. Let $0<\lambda \leq 1$. Since for any polynomial $R \in \mathcal{P}_{2 n+1}$,

$$
\left|R(x)-H_{n+1}[R](x)\right|=\left|\sum_{j=1}^{n+1} R^{\prime}\left(\xi_{j, n+1}^{(\lambda)}\right) \frac{E_{n+1}^{(\lambda)}(x)}{E_{n+1}^{(\lambda)}{ }^{\prime}\left(\xi_{j, n+1}^{(\lambda)}\right)} l_{j, n+1}(x)\right|,
$$

we have using [19, (25)],

$$
\begin{aligned}
\|(R & \left.-H_{n+1}[R]\right) u \|_{L_{p}[-1,1]}^{p} \sim \int_{A_{n}}\left|\left(R(x)-H_{n+1}[R](x)\right) u(x)\right|^{p} d x \\
& \lesssim \int_{A_{n}} \sum_{j=1}^{n+1} \frac{R^{\prime}\left(\xi_{j, n+1}^{(\lambda)}\right) E_{n+1}^{(\lambda)}(x)}{E_{n+1}^{(\lambda)}{ }^{\prime}\left(\xi_{j, n+1}^{(\lambda)}\right)} l_{j, n+1}(x) \Gamma_{1}(x) u^{p}(x) d x \\
& \lesssim \sum_{j=1}^{n+1}\left|\frac{R^{\prime}\left(\xi_{j, n+1}^{(\lambda)}\right)}{E_{n+1}^{(\lambda)}{ }^{\prime}\left(\xi_{j, n+1}^{(\lambda)}\right)}\right|\left|\int_{A_{n}} E_{n+1}^{(\lambda)}(x) l_{j, n+1}(x) \Gamma_{1}(x) u^{p}(x) d x\right|,
\end{aligned}
$$

where $\Gamma_{1}(x):=\sigma_{1}(x)\left|R(x)-H_{n+1}[R](x)\right|^{p-1}, \sigma_{1}(x):=\operatorname{sgn}\left(R-H_{n+1}[R]\right)(x)$, and $A_{n}:=\left[-1+C n^{-2}, 1-C n^{-2}\right] \backslash \bigcup_{k=1}^{r-1}\left(t_{k}-\frac{a}{n}, t_{k}+\frac{a}{n}\right), a>0$ fixed. Here, if we let

$$
\Pi(t):=\int_{A_{n}} \frac{E_{n+1}^{(\lambda)}(x)\left(E_{n+1}^{(\lambda)}(x)-E_{n+1}^{(\lambda)}(t)\right)}{x-t} \Gamma_{1}(x) u^{p}(x) d x,
$$

then using [11, Theorem 2.2], we have by (3.8),

$$
\begin{aligned}
\|(R & \left.-H_{n+1}[R]\right) u \|_{L_{p}[-1,1]}^{p} \\
& \lesssim \sum_{j=1}^{n+1} \frac{\left|R^{\prime}\left(\xi_{j, n+1}^{(\lambda)}\right)\right|}{\left|E_{n+1}^{(\lambda)}{ }^{\prime}\left(\xi_{j, n+1}^{(\lambda)}\right)\right|^{2}}\left|\Pi\left(\xi_{j, n+1}^{(\lambda)}\right)\right| \\
& \lesssim \frac{\left\|R^{\prime} \varphi\right\|_{\infty}}{n} \sum_{j=1}^{n+1} \frac{\varphi^{2 \lambda-1}\left(\xi_{j, n+1}^{(\lambda)}\right)}{n^{3-2 \lambda}}\left|\Pi\left(\xi_{j, n+1}^{(\lambda)}\right)\right| \\
& \lesssim \frac{\left\|R^{\prime} \varphi\right\|_{\infty}}{n} \frac{1}{n^{2(1-\lambda)}} \int_{A_{n}}|\Pi(t)| \varphi^{2(\lambda-1)}(t) d t \\
& \leq \frac{\left\|R^{\prime} \varphi\right\|_{\infty}}{n} \frac{1}{n^{2(1-\lambda)}} \int_{A_{n}} \varphi^{2(\lambda-1)}(t)\left(\left|H\left[E_{n+1}^{(\lambda)}{ }^{2} \Gamma_{1} u^{p}\right]\right|\right. \\
& \left.\quad+\left|E_{n+1}(t)\right|\left|H\left[E_{n+1}^{(\lambda)} \Gamma_{1} u^{p}\right]\right|\right) d t \\
& \frac{\left\|R^{\prime} \varphi\right\|_{\infty}}{n}\left(I_{1}+I_{2}\right),
\end{aligned}
$$


where $H$ is the Hilbert transform. By (3.2), (3.22), and Hölder inequality, we obtain

$$
\begin{aligned}
\left|I_{1}\right| & \lesssim \frac{1}{n^{2(1-\lambda)}} \int_{A_{n}}\left(E_{n+1}^{(\lambda)}(t)\right)^{2}\left|\Gamma_{1}(t) u^{p}\right|\left|H\left[g_{1} \varphi^{2(\lambda-1)}\right](t)\right| d t \\
& \left(g_{1}:=\operatorname{sgn} H\left[\left(E_{n+1}^{(\lambda)}\right)^{2} \Gamma_{1}\right]\right) \\
& \lesssim \int_{A_{n}} u^{p} \varphi^{2(1-\lambda)}\left|\Gamma_{1}\right|\left|H\left[g_{1} \varphi^{2(\lambda-1)}\right]\right| d t \\
& \lesssim\left\|u \varphi^{2(1-\lambda)} H\left[g_{1} \varphi^{2(\lambda-1)}\right]\right\|_{L_{p}\left(A_{n}\right)}\left\|\Gamma_{1} u^{p-1}\right\|_{L_{q}\left(A_{n}\right)} \\
& \lesssim\left\|\left(R(x)-H_{n+1}[R](x)\right) u\right\|_{L_{p}\left(A_{n}\right)}^{p-1},
\end{aligned}
$$

because $\left\|u \varphi^{2(1-\lambda)} H\left[g_{1} \varphi^{2(\lambda-1)}\right]\right\|_{L_{p}\left(A_{n}\right)}<\infty$ by [19, p. 676]. Similarly, we have

$$
\begin{aligned}
\left|I_{2}\right| & \leq \frac{1}{n^{2(1-\lambda)}} \int_{A_{n}} \varphi^{2(\lambda-1)}\left|E_{n+1}^{(\lambda)}(t) \| H\left[E_{n+1} \Gamma_{1} u^{p}\right]\right| d t \\
& \lesssim \int_{A_{n}} \frac{\varphi^{\lambda-1}(t)}{n^{(1-\lambda)}}\left|H\left[E_{n+1}^{(\lambda)} \Gamma_{1} u^{p}\right]\right| d t \\
& \lesssim \int_{A_{n}} \frac{\left|E_{n+1}^{(\lambda)} \Gamma_{1}(t)\right| u^{p}}{n^{(1-\lambda)}} H\left[g_{2} \varphi^{\lambda-1}\right] d t \quad\left(g_{2}(t):=\operatorname{sgn} H\left[E_{n+1}^{(\lambda)} \Gamma_{1}\right]\right) \\
& \lesssim \int_{A_{n}} u \varphi^{1-\lambda}(t) H\left[g_{2} \varphi^{\lambda-1}\right] \Gamma_{1}(t) u^{p-1} d t \\
& \left.\lesssim\left\|u \varphi^{1-\lambda}(t) H\left[g_{2} \varphi^{\lambda-1}\right]\right\|\right|_{L_{p}\left(A_{n}\right)}\left\|\Gamma_{1} u^{p-1}\right\|_{L_{q}\left(A_{n}\right)} \\
& \lesssim\left\|\left(R(x)-H_{n+1}[R](x)\right) u(x)\right\|_{L_{p}\left(A_{n}\right)}^{p} .
\end{aligned}
$$

Thus, we have for $0<\lambda \leq 1$,

$$
\left\|\left(R-H_{n+1}[R]\right) u\right\|_{L_{p}[-1,1]} \lesssim \frac{\left\|R^{\prime} \varphi\right\|_{\infty}}{n} .
$$

Now, let $\lambda=0$. Then for any polynomial $R \in \mathcal{P}_{2 n+1}$,

$$
\begin{aligned}
& \left|R(x)-H_{n+1}[R](x)\right|=\left|\sum_{j=1}^{n+1} R^{\prime}\left(\xi_{j, n+1}^{(\lambda)}\right) \frac{E_{n+1}^{(\lambda)}(x)}{E_{n+1}^{(\lambda)}{ }^{\prime}\left(\xi_{j, n+1}^{(\lambda)}\right)} l_{j, n+1}(x)\right| \\
& \leq\left|\sum_{j=2}^{n} R^{\prime}\left(\xi_{j, n+1}^{(\lambda)}\right) \frac{E_{n+1}^{(\lambda)}(x)}{E_{n+1}^{(\lambda)}{ }^{\prime}\left(\xi_{j, n+1}^{(\lambda)}\right)} l_{j, n+1}(x)\right| \\
& +\sum_{j=1, n+1}\left|R^{\prime}\left(\xi_{j, n+1}^{(\lambda)}\right)\right| \frac{\left|E_{n+1}^{(\lambda)}(x)\right|}{\left|E_{n+1}^{(\lambda)}{ }^{\prime}\left(\xi_{j, n+1}^{(\lambda)}\right)\right|}\left|l_{j, n+1}(x)\right| \\
& =: \quad K_{1}(x)+K_{2}(x)
\end{aligned}
$$

and

$$
\left\|\left(R-H_{n+1}[R]\right) u\right\|_{L_{p}[-1,1]} \lesssim\left\|K_{1} u\right\|_{L_{p}[-1,1]}+\left\|K_{2} u\right\|_{L_{p}[-1,1]} .
$$

We have by the same reason as in the proof of Lemma 3.8

$$
\left\|K_{2} u\right\|_{L_{p}[-1,1]} \lesssim\left\|K_{2}\right\|_{\infty}\|u\|_{L_{p}[-1,1]} \lesssim \frac{\left\|R^{\prime} \varphi\right\|_{\infty}}{n},
$$


and we obtain in the same way as in the case $0<\lambda \leq 1$

$$
\left\|K_{1} u\right\|_{L_{p}[-1,1]} \lesssim \frac{\left\|R^{\prime} \varphi\right\|_{\infty}}{n}
$$

Therefore, we proved (3.23) for $0 \leq \lambda \leq 1$.

Proof of Theorem 2.3. Since $u \in L_{p}$ and for any polynomial $R \in \mathcal{P}_{2 n+1}$ by (3.23)

$$
\begin{aligned}
& \left\|\left(H_{n+1}[f]-f\right) u\right\|_{p} \\
\leq & \|(f-R) u\|_{p}+\left\|\left(H_{n+1}[f-R]\right) u\right\|_{p}+\left\|\left(H_{n+1}[R]-R\right) u\right\|_{p} \\
\lesssim & \|f-R\|_{\infty}\|u\|_{p}+\frac{\left\|R^{\prime} \varphi\right\|_{\infty}}{n},
\end{aligned}
$$

we have from [2, Theorem 7.3.1],

$$
\left\|\left(H_{n+1}[f]-f\right) u\right\|_{p} \lesssim \omega_{\varphi, \infty}\left(f, \frac{1}{n}\right) .
$$

3.2. Convergence of the Hermite-Fejér interpolation polynomials for $F_{2 n+1}^{(\lambda)}(x)$. In this subsection, we prove Theorems 2.2 and 2.4 and we will omit the details because the most methods are similar to Subsection 3.1.

Lemma 3.10. Let $\lambda \in[0,1]$. Then we have for large $n>0$,

$$
\left|\mathcal{H}_{2,2 n+1}[f](x)\right| \lesssim\|f\|_{\infty}\left(\varphi^{2(1-2 \lambda)}(x)+1\right) .
$$

Proof. Let $0<\lambda \leq 1$. It is sufficient to prove (3.15) for $0 \leq x \leq 1-\frac{a}{n^{2}}$, where $a>0$ is a fixed, sufficiently small constant. In the following let $\|f\|_{\infty}=1$ and let $x \in\left[y_{k-1,2 n+1}^{(\lambda)}, y_{k+1,2 n+1}^{(\lambda)}\right] \cap\left[0,1-\frac{a}{n^{2}}\right]\left(y_{0,2 n+1}^{(\lambda)}=-1, y_{2 n+2,2 n+1}^{(\lambda)}:=1\right)$ for some $1 \leq k \leq 2 n+1$. Then,

$$
\begin{aligned}
\left|\mathcal{H}_{2,2 n+1}[f](x)\right| & \lesssim \sum_{j=1}^{2 n+1}\left|\frac{F_{2 n+1}^{(\lambda)}{ }^{\prime \prime}\left(y_{j, 2 n+1}^{(\lambda)}\right)}{F_{2 n+1}^{(\lambda)}{ }^{\prime(\lambda)}\left(y_{j, 2 n+1}^{(\lambda)}\right)}\right|\left|x-y_{j, 2 n+1}^{(\lambda)}\right| l_{j, 2 n+1}^{2}(x) \\
& =\sum_{j \in[k-2, k+2]}+\sum_{j \notin[k-2, k+2]}:=J_{1}+J_{2} .
\end{aligned}
$$

Since we have by (3.1), (3.3), (3.7), and (3.9), similar to (3.16),

$$
\left|l_{j, 2 n+1}(x)\right| \sim 1, \quad j \in[k-2, k+2] \cap[1,2 n+1],
$$

we have by (3.1), (3.7), (3.9), and (3.25),

$$
J_{1} \lesssim \frac{n^{1+\lambda} \varphi^{-2-\lambda}(x)}{n \varphi^{-2}(x)} \frac{\varphi(x)}{n} \lesssim n^{-1+\lambda} \varphi^{-1+\lambda}(x) \lesssim 1 .
$$

For $J_{2}$, we first assume $0 \leq x \leq y_{2 n-1,2 n+1}^{(\lambda)}$. Then we have by (3.1), (3.3), (3.9), and (3.11),

$$
\begin{aligned}
J_{2} & \lesssim \frac{\varphi^{2(1-2 \lambda)}(x)}{n^{1-\lambda}} \sum_{j \notin[k-2, k+2]} \frac{\varphi^{5 \lambda-2}\left(y_{j, 2 n+1}^{(\lambda)}\right)}{n} \frac{1}{\left|x-y_{j, 2 n+1}^{(\lambda)}\right|} \\
& \lesssim \frac{\varphi^{2(1-2 \lambda)}(x)}{n^{1-\lambda}} \int_{\left[-1+\frac{C}{n^{2}}, 1-\frac{C}{n^{2}}\right] \backslash\left[y_{k-2,2 n+1}^{(\lambda)}, y_{k+2,2 n+1}^{(\lambda)}\right]} \frac{\varphi^{5 \lambda-3}(t)}{|x-t|} d t .
\end{aligned}
$$


We assume $0<x \leq 1-\frac{a}{n^{2}}$. Let $\lambda \in(0,1 / 2]$. Then

$$
\int \frac{\varphi^{5 \lambda-3}(t)}{|x-t|} d t \lesssim \int_{0}^{x_{*}}+\int_{x^{*}}^{\frac{1+x^{*}}{2}}+\int_{\frac{1+x^{*}}{2}}^{1-\frac{C}{n^{2}}} \frac{\varphi^{5 \lambda-3}(t)}{|x-t|} d t .
$$

Since $\varphi^{2(2 \lambda-1)}(x)$ and $\varphi^{\lambda-1}(x)$ are increasing on $(0,1)$,

$$
\begin{gathered}
\left|\int_{0}^{x_{*}} \frac{\varphi^{5 \lambda-3}(t)}{x-t} d t\right| \lesssim \varphi^{2(2 \lambda-1)}\left(x_{*}\right)\left|\int_{0}^{x_{*}} \frac{\varphi^{\lambda-1}(t)}{x-t} d t\right| \lesssim \varphi^{4 \lambda-2}(x) n^{1-\lambda}, \\
\int_{x^{*}}^{\frac{1+x^{*}}{2}} \frac{\varphi^{5 \lambda-3}(t)}{t-x} d t \lesssim \varphi^{2(2 \lambda-1)}\left(\frac{1+x^{*}}{2}\right) \int_{x^{*}}^{\frac{1+x^{*}}{2}} \frac{\varphi^{\lambda-1}(t)}{t-x} d t \lesssim \varphi^{4 \lambda-2}(x) n^{1-\lambda},
\end{gathered}
$$

and since $|x-t| \gtrsim|1-t|$ for $\frac{1+x^{*}}{2}<t<1-\frac{C}{n^{2}}$,

$$
\begin{aligned}
\int_{\frac{1+x^{*}}{2}}^{1-\frac{C}{n^{2}}} \frac{\varphi^{5 \lambda-3}(t)}{|x-t|} d t & \lesssim \frac{1}{\left|\frac{1+x^{*}}{2}-x\right|^{1-2 \lambda}} \int_{\frac{1+x^{*}}{2}}^{1-\frac{C}{n^{2}}} \frac{1}{|x-t|^{2 \lambda}(1-t)^{\frac{3}{2}-\frac{5 \lambda}{2}}} d t \\
& \lesssim \varphi^{-2(1-2 \lambda)}(x) \int_{\frac{1+x^{*}}{2}}^{1-\frac{C}{n^{2}}} \frac{1}{(1-t)^{\frac{3}{2}-\frac{\lambda}{2}}} d t \lesssim \varphi^{4 \lambda-2}(x) n^{1-\lambda} .
\end{aligned}
$$

Thus, we have $J_{2} \lesssim 1$ for $\lambda \in(0,1 / 2]$. Next, let $\lambda \in(1 / 2,1]$. Then since $\varphi^{4 \lambda-2}(x)$ is bounded on $(0,1)$, we have by the above estimation,

$$
\int \frac{\varphi^{5 \lambda-3}(t)}{|x-t|} d t \lesssim \int_{0}^{x_{*}}+\int_{x^{*}}^{\frac{1+x^{*}}{2}}+\int_{\frac{1+x^{*}}{2}}^{1-\frac{C}{n^{2}}} \frac{\varphi^{\lambda-1}(t)}{|x-t|} d t \lesssim n^{1-\lambda} .
$$

So, for $\lambda \in(1 / 2,1]$, we also have $J_{2} \lesssim \varphi^{2(1-2 \lambda)}(x)$. For $y_{2 n-1,2 n+1}^{(\lambda)}<x \leq 1$ we have by the same reason as the above case

$$
J_{2} \lesssim \frac{\varphi^{2(1-2 \lambda)}(x)}{n^{1-\lambda}} \int_{0}^{x_{*}} \frac{\varphi^{5 \lambda-3}(t)}{|x-t|} d t \lesssim \begin{cases}1, & \lambda \in(0,1 / 2], \\ \varphi^{2(1-2 \lambda)}(x), & \lambda \in(1 / 2,1] .\end{cases}
$$

Therefore, we have for $\lambda \in(0,1]$,

$$
\left|\mathcal{H}_{2,2 n+1}[f](x)\right| \lesssim\|f\|_{\infty}\left(\varphi^{2(1-2 \lambda)}(x)+1\right) .
$$

Now, we consider the case of $\lambda=0$. Then

$$
\begin{aligned}
\left|\mathcal{H}_{2,2 n+1}[f](x)\right| & \lesssim \sum_{j=2}^{2 n}+\sum_{j=1,2 n+1}\left|\frac{F_{2 n+1}^{(\lambda)}{ }^{\prime \prime}\left(y_{j, 2 n+1}^{(\lambda)}\right)}{F_{2 n+1}^{(\lambda)}{ }^{\prime}\left(y_{j, 2 n+1}^{(\lambda)}\right)}\right|\left|x-y_{j, 2 n+1}^{(\lambda)}\right| l_{j, 2 n+1}^{2}(x) \\
=: & K_{1}+K_{2},
\end{aligned}
$$

and we have $K_{2} \lesssim 1$ and $K_{1} \lesssim 1$ by (3.5), (3.9), (3.12) and by the same way as in the case $0<\lambda \leq 1$. Therefore, (3.24) is proved for $0 \leq \lambda \leq 1$.

Lemma 3.11. Let $\lambda \in(0,1]$. Then we have for large $n>0$,

$$
\sum_{\nu=1}^{2 n+1} \varphi^{2 \lambda-1}\left(y_{\nu, 2 n+1}^{(\lambda)}\right) \varphi^{1-2 \lambda}(x)\left|l_{\nu, 2 n+1}(x)\right| \lesssim\left(\varphi^{2(1-2 \lambda)}(x)+1\right) \log n .
$$

In the case of $\lambda=0$, we have

$$
\sum_{\nu=2}^{2 n} \varphi^{-1}\left(y_{\nu, 2 n+1}^{(0)}\right) \varphi(x)\left|l_{\nu, 2 n+1}(x)\right| \lesssim\left(\varphi^{2}(x)+1\right) \log n .
$$


Proof. Let $0<\lambda \leq 1$. Let $x \in\left[y_{k-1,2 n+1}^{(\lambda)}, y_{k+1,2 n+1}^{(\lambda)}\right]$ and split the left side of (3.26) into $J_{1}$ and $J_{2}$ :

$$
\sum_{\nu \in[k-2, k+2]}+\sum_{\nu \notin[k-2, k+2]}\left|l_{\nu, 2 n+1}(x)\right| \varphi^{1-2 \lambda}(x) \varphi^{2 \lambda-1}\left(y_{\nu, 2 n+1}^{(\lambda)}\right)=: J_{1}+J_{2} .
$$

Then, we have by (3.25) and (3.1),

$$
J_{1} \lesssim \sum_{\nu \in[k-2, k+2] \cap[1,2 n+1]} \varphi^{1-2 \lambda}(x) \varphi^{2 \lambda-1}\left(y_{\nu, 2 n+1}^{(\lambda)}\right) \lesssim 1,
$$

and we have by (3.3) and (3.9)

$$
\begin{aligned}
J_{2} & \lesssim \sum_{\nu \notin[k-2, k+2]} \frac{\left|F_{2 n+1}^{(\lambda)}(x)\right| \varphi^{1-2 \lambda}(x) \varphi^{2 \lambda-1}\left(y_{\nu, 2 n+1}^{(\lambda)}\right)}{\left|F_{2 n+1}^{(\lambda)}{ }^{\prime}\left(y_{\nu, 2 n+1}^{(\lambda)}\right)\right|}\left|\frac{1}{x-y_{\nu, 2 n+1}^{(\lambda)}}\right| \\
& \lesssim \varphi^{2(1-2 \lambda)}(x) \sum \frac{\varphi^{4 \lambda-1}\left(y_{\nu, 2 n+1}^{(\lambda)}\right)}{n\left|x-y_{\nu, 2 n+1}^{(\lambda)}\right|} \\
& \lesssim \varphi^{2(1-2 \lambda)}(x) \int_{[-1,1] \backslash\left[y_{k-2,2 n+1}^{(\lambda)}, y_{k+2,2 n+1}^{(\lambda)}\right]} \frac{\varphi^{4 \lambda-2}(t)}{|x-t|} d t \\
& \lesssim \varphi^{2(1-2 \lambda)}(x)\left(\varphi^{2(2 \lambda-1)}(x)+1\right) \log n \lesssim\left(1+\varphi^{2(1-2 \lambda)}(x)\right) \log n .
\end{aligned}
$$

This is because for $0<\lambda \leq 1 / 2$, since $\varphi^{4 \lambda-2}(t)$ is increasing on $(0,1)$, similar to (3.19),

$$
\int_{[-1,1] \backslash\left[y_{k-2,2 n+1}^{(\lambda)}, y_{k+2,2 n+1}^{(\lambda)}\right]} \frac{\varphi^{4 \lambda-2}(t)}{|x-t|} d t \lesssim \varphi^{4 \lambda-2}(x) \log n,
$$

and for $1 / 2<\lambda \leq 1$, since $\varphi^{4 \lambda-2}(t)$ is bounded on $(0,1)$,

$$
\int_{[-1,1] \backslash\left[y_{k-2,2 n+1}^{(\lambda)}, y_{k+2,2 n+1}^{(\lambda)}\right]} \frac{\varphi^{4 \lambda-2}(t)}{|x-t|} d t \lesssim \int_{[-1,1] \backslash\left[y_{k-2,2 n+1}^{(\lambda)}, y_{k+2,2 n+1}^{(\lambda)}\right]} \frac{1}{|x-t|} d t \lesssim \log n .
$$

For $\lambda=0$, the proof is similar to the case of $0<\lambda \leq 1$ by (3.5) and (3.9). Therefore, (3.27) is also proved.

Lemma 3.12. Let $\lambda \in[0,1]$. For any polynomial $R \in \mathcal{P}_{4 n+1}$,

$$
\left|R(x)-\mathcal{H}_{2 n+1}[R](x)\right| \lesssim\left\|R^{\prime} \varphi\right\|_{\infty}\left(\varphi^{2(1-2 \lambda)}(x)+1\right) \frac{\log n}{n} .
$$

Proof. Let $0<\lambda \leq 1$. For any polynomial $R \in \mathcal{P}_{4 n+1}$ by (2.4), (3.3), (3.9), and (3.26),

$$
\begin{aligned}
\mid R(x) & -\mathcal{H}_{2 n+1}[R](x) \mid \\
& \lesssim \frac{\left\|R^{\prime} \varphi\right\|_{\infty}}{n} \sum_{\nu=1}^{2 n+1} \frac{\varphi^{1-2 \lambda}(x) \varphi^{2 \lambda-1}\left(y_{\nu, 2 n+1}^{(\lambda)}\right)}{n}\left|l_{\nu, 2 n+1}(x)\right| \\
& \lesssim \frac{\left\|R^{\prime} \varphi\right\|_{\infty}}{n} \log n\left(\varphi^{2(1-2 \lambda)}(x)+1\right) .
\end{aligned}
$$


Now, we consider the case of $\lambda=0$. Then

$$
\begin{aligned}
\left|R(x)-H_{n+1}[R](x)\right| & \leq \sum_{j=1}^{2 n+1}\left|\frac{F_{2 n+1}^{(0)}(x)}{F_{2 n+1}^{(0)}(x)^{\prime}\left(y_{j, 2 n+1}^{(0)}\right)}\right|\left|l_{j, 2 n+1}(x)\right|\left|R^{\prime}\left(y_{j, 2 n+1}^{(0)}\right)\right| \\
& =\sum_{j=2}^{2 n}+\sum_{j=1,2 n+1}\left|\frac{F_{2 n+1}^{(0)}(x)}{F_{2 n+1}^{(0)}(x)^{\prime}\left(y_{j, 2 n+1}^{(0)}\right)}\right|\left|l_{j, 2 n+1}(x)\right|\left|R^{\prime}\left(y_{j, 2 n+1}^{(0)}\right)\right| \\
& =: K_{1}+K_{2} .
\end{aligned}
$$

By (3.5), (3.9), and [19, (26)] we have

$$
K_{2} \lesssim \frac{\left\|R^{\prime} \varphi\right\|_{\infty}}{n},
$$

and in the same way as in the case $0<\lambda \leq 1$, we obtain

$$
K_{1} \lesssim \frac{\left\|R^{\prime} \varphi\right\|_{\infty}}{n} \log n\left(\varphi^{2(1-2 \lambda)}(x)+1\right) .
$$

Therefore, we have (3.28) for $0 \leq \lambda \leq 1$.

Proof of Theorem 2.2. Since we have by (3.14) and (3.24)

$$
\left|\mathcal{H}_{2 n+1}[f](x)\right| \lesssim\|f\|_{\infty}\left(\varphi^{2(1-2 \lambda)}(x)+1\right)
$$

(i.e., $\left.\left\|\mathcal{H}_{2 n+1}\right\|_{\infty}=O(1), 0<\lambda \leq 1 / 2\right)$ and for given $\epsilon>0$, there exists a polynomial $R$ such that for $x \in[-1,1]$

$$
|f(x)-R(x)|<\epsilon,
$$

if $0<\lambda \leq 1 / 2$, we have by (3.28)

$$
\lim _{n \rightarrow \infty}\left|\mathcal{H}_{2 n+1}[f](x)-f(x)\right| \lesssim\|f-P\|_{\infty}+\lim _{n \rightarrow \infty}\left\|R^{\prime} \varphi\right\|_{\infty} \frac{\log n}{n} \lesssim \epsilon,
$$

and if $1 / 2<\lambda<1$, we have by (3.28)

$$
\begin{aligned}
& \lim _{n \rightarrow \infty}\left|\left(\mathcal{H}_{2 n+1}[f](x)-f(x)\right) \varphi^{2(2 \lambda-1)}(x)\right| \\
& \quad \lesssim\|f(x)-R(x)\|_{\infty}+\lim _{n \rightarrow \infty}\left\|R^{\prime} \varphi\right\|_{\infty} \frac{\log n}{n} \lesssim \epsilon .
\end{aligned}
$$

Therefore, (2.10) and (2.12) are proved since $\epsilon>0$ is arbitrary. Moreover, since for any polynomial $R \in \mathcal{P}_{\left[\frac{n}{\log n}\right]}$

$$
\left|\mathcal{H}_{2 n+1}[f](x)-f(x)\right| \lesssim\left(\|f-R\|_{\infty}+\left\|R^{\prime} \varphi\right\|_{\infty} /\left[\frac{n}{\log n}\right]\right)\left(\varphi^{2(1-2 \lambda)}(x)+1\right),
$$

we have from [2, Theorem 7.3.1]

$$
\left|\mathcal{H}_{2 n+1}[f](x)-f(x)\right| \lesssim \omega_{\varphi, \infty}\left(f, \frac{\log n}{n}\right)\left(\varphi^{2(1-2 \lambda)}(x)+1\right) .
$$

Also, since for any polynomial $R \in \mathcal{P}_{4 n+1}$

$$
\left|\mathcal{H}_{2 n+1}[f](x)-f(x)\right| \lesssim\left(\|f-R\|_{\infty}+\left\|R^{\prime} \varphi\right\|_{\infty} \frac{\log n}{n}\right)\left(\varphi^{2(1-2 \lambda)}(x)+1\right),
$$

we have

$$
\left|\mathcal{H}_{2 n+1}[f](x)-f(x)\right| \lesssim \omega_{\varphi, \infty}\left(f, \frac{1}{n}\right) \log n\left(\varphi^{2(1-2 \lambda)}(x)+1\right) .
$$


Therefore, (2.11) and (2.13) are proved.

Lemma 3.13. Let $\lambda \in[0,1], 1<p<\infty$, and let $u\left(\varphi^{2(1-2 \lambda)}(x)+1\right) \in L_{p}$. Then for any polynomial $R \in \mathcal{P}_{4 n+1}$,

$$
\left\|\left(R-\mathcal{H}_{2 n+1}[R]\right) u\right\|_{L_{p}[-1,1]} \lesssim \frac{\left\|R^{\prime} \varphi\right\|_{L_{\infty}}}{n} .
$$

Proof. Let $0<\lambda \leq 1$. For any polynomial $R \in \mathcal{P}_{4 n+1}$,

$$
\begin{aligned}
\|(R & \left.-\mathcal{H}_{2 n+1}[R]\right) u \|_{L_{p}[-1,1]}^{p} \\
& \lesssim \sum_{\nu=1}^{2 n+1}\left|\frac{R^{\prime}\left(y_{\nu, 2 n+1}^{(\lambda)}\right)}{F_{2 n+1}^{(\lambda)}{ }^{\prime}\left(y_{\nu, 2 n+1}^{(\lambda)}\right)}\right|\left|\int_{A_{n}} F_{2 n+1}^{(\lambda)}(x) l_{\nu, 2 n+1}(x) \Gamma_{2}(x) u^{p}(x) d x\right|,
\end{aligned}
$$

where $\Gamma_{2}(x):=\sigma_{2}(x)\left|R(x)-\mathcal{H}_{2 n+1}[R](x)\right|^{p-1}$ and $\sigma_{2}(x):=\operatorname{sgn}\left(R-\mathcal{H}_{2 n+1}[R]\right)(x)$. Here, if we let

$$
\Pi(t):=\int_{A_{n}} \frac{F_{2 n+1}^{(\lambda)}(x)\left(F_{2 n+1}^{(\lambda)}(x)-F_{2 n+1}^{(\lambda)}(t)\right)}{x-t} \Gamma_{2}(x) u^{p}(x) d x,
$$

then we have

$$
\begin{aligned}
\| & \left(R-\mathcal{H}_{2 n+1}[R]\right) u \|_{L_{p}[-1,1]}^{p} \\
& \lesssim \frac{\left\|R^{\prime} \varphi\right\|_{\infty}}{n} \int_{A_{n}}|\Pi(t)| \varphi^{2(2 \lambda-1)}(t) d t \\
& \lesssim \frac{\left\|R^{\prime} \varphi\right\|_{\infty}}{n} \int_{A_{n}} \varphi^{2(2 \lambda-1)}(t)\left(\left|H\left[\left(F_{2 n+1}^{(\lambda)}\right)^{2} \Gamma_{2} u^{p}\right]\right|+\left|F_{2 n+1}(t) \| H\left[F_{2 n+1}^{(\lambda)} \Gamma_{2} u^{p}\right]\right|\right) d t \\
& =: \frac{\left\|R^{\prime} \varphi\right\|_{\infty}}{n}\left(I_{1}+I_{2}\right) .
\end{aligned}
$$

Since $\left\|u \varphi^{2(1-2 \lambda)} H\left[g_{3} \varphi^{2(2 \lambda-1)}\right]\right\|_{L_{p}\left(A_{n}\right)}<\infty$ by [19, p. 676], we have

$$
\begin{aligned}
\left|I_{1}\right| & \lesssim \int_{A_{n}} u \varphi^{2(1-2 \lambda)}\left|\Gamma_{2} u^{p-1} \| H\left[g_{3} \varphi^{2(2 \lambda-1)}\right]\right| d t \\
& \lesssim\left\|u \varphi^{2(1-2 \lambda)} H\left[g_{3} \varphi^{2(2 \lambda-1)}\right]\right\|_{L_{p}\left(A_{n}\right)}\left\|\Gamma_{2} u^{p-1}\right\|_{L_{q}\left(A_{n}\right)} \\
& \lesssim\left\|\left(R-\mathcal{H}_{2 n+1}[R]\right) u\right\|_{L_{p}\left(A_{n}\right)}^{p-1},
\end{aligned}
$$

where $g_{3}:=\operatorname{sgn} H\left[\left(F_{2 n+1}^{(\lambda)}\right)^{2} \Gamma_{2} u^{p}\right]$. Similarly,

$$
\begin{aligned}
\left|I_{2}\right| & \lesssim\left\|u \varphi^{1-2 \lambda}(t) H\left[g_{4} \varphi^{2 \lambda-1}\right]\right\|_{L_{p}\left(A_{n}\right)}|| \Gamma_{2} u^{p-1} \|_{L_{q}\left(A_{n}\right)} \\
& \lesssim\left\|\left(R-\mathcal{H}_{2 n+1}[R]\right) u\right\|_{L_{p}\left(A_{n}\right)}^{p-1},
\end{aligned}
$$

where $g_{4}(t):=\operatorname{sgn} H\left[F_{2 n+1} \Gamma_{2} u^{p}\right]$. Then we have for $0<\lambda \leq 1$

$$
\left\|\left(R-\mathcal{H}_{2 n+1}[R]\right) u\right\|_{L_{p}[-1,1]} \lesssim \frac{\left\|R^{\prime} \varphi\right\|_{\infty}}{n} .
$$


Now, let $\lambda=0$. Then for any polynomial $R \in \mathcal{P}_{4 n+1}$,

$$
\begin{aligned}
\left|R(x)-\mathcal{H}_{2 n+1}[R](x)\right| & =\left|\sum_{j=1}^{2 n+1} R^{\prime}\left(y_{j, 2 n+1}^{(0)}\right) \frac{F_{2 n+1}^{(0)}(x)}{F_{2 n+1}^{(0)}{ }^{\prime}\left(y_{j, 2 n+1}^{(0)}\right)} l_{j, 2 n+1}(x)\right| \\
& \leq\left|\sum_{j=2}^{2 n} R^{\prime}\left(y_{j, 2 n+1}^{(0)}\right) \frac{F_{2 n+1}^{(0)}(x)}{F_{2 n+1}^{(0)}{ }^{\prime}\left(y_{j, 2 n+1}^{(0)}\right)} l_{j, 2 n+1}(x)\right| \\
& +\sum_{j=1,2 n+1}\left|R^{\prime}\left(y_{j, 2 n+1}^{(0)}\right)\right| \frac{\left|F_{2 n+1}^{(0)}(x)\right|}{\left|F_{2 n+1}^{(0)}{ }^{\prime}\left(y_{j, 2 n+1}^{(0)}\right)\right|}\left|l_{j, 2 n+1}(x)\right| \\
& =: K_{1}(x)+K_{2}(x)
\end{aligned}
$$

and

$$
\left\|\left(R-H_{n+1}[R]\right) u\right\|_{L_{p}[-1,1]} \lesssim\left\|K_{1} u\right\|_{L_{p}[-1,1]}+\left\|K_{2} u\right\|_{L_{p}[-1,1]} .
$$

We have by the same reason as in the proof of Lemma 3.12

$$
\left\|K_{2} u\right\|_{L_{p}[-1,1]} \lesssim\left\|K_{2}\right\|_{\infty}\|u\|_{L_{p}[-1,1]} \lesssim \frac{\left\|R^{\prime} \varphi\right\|_{\infty}}{n},
$$

and we obtain in the same way as in the case $0<\lambda \leq 1$

$$
\left\|K_{1} u\right\|_{L_{p}[-1,1]} \lesssim \frac{\left\|R^{\prime} \varphi\right\|_{\infty}}{n} .
$$

Therefore, we proved (3.23) for $0 \leq \lambda \leq 1$.

Proof of Theorem 2.4. Since $u\left(\varphi^{2(1-2 \lambda)}(x)+1\right) \in L_{p}$, we have

$$
\begin{aligned}
\left\|\left(\mathcal{H}_{2 n+1}[f]-f\right) u\right\|_{p} \\
\quad \leq\|(f-R) u\|_{p}+\left\|\left(\mathcal{H}_{2 n+1}[f-R]\right) u\right\|_{p}+\left\|\left(\mathcal{H}_{2 n+1}[R]-R\right) u\right\|_{p} \\
\quad \lesssim\|f-R\|_{\infty}\|u\|_{p}+\frac{\left\|R^{\prime} \varphi\right\|_{\infty}}{n} \\
\quad \lesssim \omega_{\varphi}\left(f, \frac{1}{n}\right) .
\end{aligned}
$$

3.3. Convergence of the Hermite interpolation polynomials for $E_{n+1}^{(\lambda)}(x)$ and $F_{2 n+1}^{(\lambda)}(x)$.

Proof of Theorems 2.5 and 2.7, Since

$$
\hat{H}_{n+1}[f]=H_{n+1}[f]+\hat{H}_{n+1}[f]-H_{n+1}[f],
$$

we have by (3.21) and by the same reason as (3.20),

$$
\begin{aligned}
\left\|\hat{H}_{n+1}[f]\right\|_{\infty} & \leq\left\|H_{n+1}[f]\right\|_{\infty}+\left\|\hat{H}_{n+1}[f]-H_{n+1}[f]\right\|_{\infty} \\
& \lesssim\|f\|_{\infty}+\frac{\left\|f^{\prime} \varphi\right\|_{\infty} \log n}{n} .
\end{aligned}
$$

From [10, Theorem 2, p. 172], there exists a polynomial $R_{n} \in \mathcal{P}_{n}$ such that

$$
\left\|\left(f^{(j)}-R_{n}^{(j)}\right) \varphi^{j}\right\|_{\infty} \lesssim \frac{\mathcal{E}_{n-N, \infty}\left(f^{(N)}\right)}{n^{N-j}} \quad \forall f \in C^{N}[-1,1], 0 \leq j \leq N .
$$


Then for this polynomial $R \in \mathcal{P}_{2 n+1}$, we have by Markov inequality and (3.30),

$$
\begin{aligned}
\left\|\left(\hat{H}_{n+1}^{(j)}[f]-f^{(j)}\right) \varphi^{j}\right\|_{\infty} \\
\quad \lesssim\left\|\left(\hat{H}_{n+1}^{(j)}[f-R]-(f-R)^{(j)}\right) \varphi^{j}\right\|_{\infty} \\
\quad \lesssim\left\|\hat{H}_{n+1}^{(j)}[f-R] \varphi^{j}\right\|_{\infty}+\left\|(f-R)^{(j)} \varphi^{j}\right\|_{\infty} \\
\quad \lesssim n^{j}\left\|\hat{H}_{n+1}[f-R]\right\|_{\infty}+\left\|(f-R)^{(j)} \varphi^{j}\right\|_{\infty} \\
\quad \lesssim n^{j}\|f-R\|_{\infty}+\frac{\left\|\left(f^{\prime}-R^{\prime}\right) \varphi\right\|_{\infty} \log n}{n^{1-j}}+\left\|(f-R)^{(j)} \varphi^{j}\right\|_{\infty} \\
\quad \lesssim n^{j} \frac{\mathcal{E}_{2 n+1-N, \infty}\left(f^{(N)}\right)}{n^{N}}+\frac{\mathcal{E}_{2 n+1-N, \infty}\left(f^{(N)}\right) \log n}{n^{N-j}}+\frac{\mathcal{E}_{2 n+1-N, \infty}\left(f^{(N)}\right)}{n^{N-j}} \\
\quad \lesssim \frac{\mathcal{E}_{2 n+1-N, \infty}\left(f^{(N)}\right) \log n}{n^{N-j}} .
\end{aligned}
$$

Since by (3.21) and by the same reason as (3.23),

$$
\begin{aligned}
\left\|\hat{H}_{n+1}[f]\right\|_{p} & \leq\left\|H_{n+1}[f]\right\|_{p}+\left\|\hat{H}_{n+1}[f]-H_{n+1}[f]\right\|_{p} \\
& \lesssim\|f\|_{\infty}+\frac{\left\|f^{\prime} \varphi\right\|_{\infty}}{n},
\end{aligned}
$$

we similarly have

$$
\left\|\left(\hat{H}_{n+1}^{(j)}[f]-f^{(j)}\right) \varphi^{(j)}\right\|_{p} \lesssim \frac{\mathcal{E}_{2 n+1-N, \infty}\left(f^{(N)}\right)}{n^{N-j}} .
$$

Proof of Theorems 2.6 and 2.8, Let

$$
v(x)= \begin{cases}1, & 0 \leq \lambda \leq 1 / 2 \\ \varphi^{2(2 \lambda-1)}(x), & 1 / 2<\lambda \leq 1\end{cases}
$$

Since

$$
\begin{aligned}
\left\|\hat{\mathcal{H}}_{2 n+1}[f] v\right\|_{\infty} & \leq\left\|\mathcal{H}_{2 n+1}[f] v\right\|_{\infty}+\left\|\left(\hat{\mathcal{H}}_{2 n+1}[f]-\mathcal{H}_{2 n+1}[f]\right) v\right\|_{\infty} \\
& \lesssim\|f\|_{\infty}+\frac{\left\|f^{\prime} \varphi\right\|_{\infty} \log n}{n}
\end{aligned}
$$

and

$$
\begin{aligned}
\left\|\hat{\mathcal{H}}_{2 n+1}[f] v\right\|_{p} & \leq\left\|\mathcal{H}_{2 n+1}[f] v\right\|_{p}+\left\|\left(\hat{\mathcal{H}}_{2 n+1}[f]-\mathcal{H}_{2 n+1}[f]\right) v\right\|_{p} \\
& \lesssim\|f\|_{\infty}+\frac{\left\|f^{\prime} \varphi\right\|_{\infty}}{n}
\end{aligned}
$$

by the same reason as in the proofs of Theorems 2.5 and 2.7, we have

$$
\left\|\left(\hat{\mathcal{H}}_{2 n+1}^{(j)}[f]-f^{(j)}\right) v \varphi^{j}\right\|_{\infty} \lesssim \frac{\mathcal{E}_{4 n+1-N, \infty}\left(f^{(N)}\right) \log n}{n^{N-j}}
$$

and

$$
\left\|\left(\hat{\mathcal{H}}_{2 n+1}^{(j)}[f]-f^{(j)}\right) v \varphi^{j}\right\|_{\infty} \lesssim \frac{\mathcal{E}_{4 n+1-N, \infty}\left(f^{(N)}\right)}{n^{N-j}} .
$$




\section{REFERENCES}

[1] P. Barrucand, Intégration Numérique, Abscisses de Kronrod-Patterson et Polynômes de Szegö, C. R. Acad. Sci. Paris, Ser. A, 270 (1970), 147-158. MR0267755 (42:2657)

[2] Z. Ditzian and V. Totik, Moduli of Smoothness, Springer Series in Computational Mathmatics, Springer-Verlag, Berlin, 9 (1987). MR0914149(89h:41002)

[3] S. Ehrich, Asymptotic properties of Stieltjes polynomials and Gauss-Kronrod quadrature formulae, J. Approx. Theory, 82 (1995), 287-303. MR.1343838 (96i:41028)

[4] S. Ehrich, Stieltjes polynomials and the error of Gauss-Kronrod quadrature formulas, in W. Gautschi, G. Golub, G. Opfer (Eds.), Applications and Computation of Orthogonal Polynomials, Proc. Conf. Oberwolfach, International Series Numerical Mathematics, 131, Birkhäuser, Basel, (1999), 57-77. MR1722715 (2000k:65047)

[5] S. Ehrich and G. Mastroianni, On the generalized Stieltjes polynomials and Lagrange interpolation, Approximation Theory and Function Series, Bolyai Soc. Math. Stud., 5 (1996), 187-203. MR1432668 (97k:41004)

[6] S. Ehrich and G. Mastroianni, Stieltjes polynomials and Lagrange interpolation, Math. Comp., 66 (1997), 311-331. MR1388888 (97j:65013)

[7] W. Gautschi, Gauss-Kronrod Quadrature - A survey, in Numerial Methods and Approximation Theory III, G.V. Milovanović, ed., Niš (1988), 39-66. MR0960329 (89k:41035)

[8] W. Gautschi and S. E. Notaris, An Algebraic and Numerical Study of Gauss-Kronrod Quadrature Formulae for Jacobi Weight Functions, Math. Comp., 51 (1988), 231-248. MR0942152 (89f:65031)

[9] H. S. Jung, Estimates for the first and second derivatives of the Stieltjes polynomials, J. Approx. Theory, 127 (2004), 155-177. MR.2058155 (2005e:42073)

[10] D. Leviatan, The behavior of the derivatives of the algebraic polynomials of best approximation, J. Approx. Theory, 35 (1982), 169-176. MR0662164 (83i:41022)

[11] D. S. Lubinsky, A. Máté and P. Nevai, Quadrature sums involving pth powers of polynomials, SIAM J. Math. Anal., 18 (1987), 531-544. MR0876290 (89h:41058)

[12] G. Monegato, A Note on Extended Gaussian Quadrature Rules, Math. Comp., 30 (1976), 812-817. MR0440878 (55:13746)

[13] G. Monegato, Positivity of Weights of Extended Gauss-Legendre Quadrature Rules, Math. Comp., 32 (1978), 243-245. MR0458809 (56:17009)

[14] G. Monegato, An Overview of Results and Questions Related to Kronrod Schemes, in Numerishe Integration, Proc. Conf. Oberwolfach, G. Hämmerlin (Ed.), ISNM 57 Birkhäuser, 1979. MR0561296 (81m:65033)

[15] G. Monegato, Stieltjes Polynomials and Related Quadrature Rules, SIAM Review, 24 (1982), 137-158. MR0652464 (83d:65067)

[16] NAG Fortran Library, Mark 15, NAG Ltd., Oxford, 1991.

[17] S. E. Notaris, Gauss-Kronrod quadrature formulae for weight functions of Bernstein-Szego type. II, J. Comput. Appl. Math., 29 (1990), 161-169. MR1041189 (91b:65030)

[18] S. E. Notaris, An overview of results on the existence or nonexistence and the error term of Gauss-Kronrod quadrature formulae, in R. V. M. Zahar (Ed.), Approximation and computation, a Festschrift in Honer of Walter Gautschi, Internat. Ser. Numer. Math., 119, Birkha̋user Basel, (1994), 485-496. MR1333638(96a:65034)

[19] P. Nevai, Mean Convergence of Lagrange Interpolation, III, Trans. Ams. Math. Soc., 282 (1984), 669-698. MR0732113 (85c:41009)

[20] P. Nevai, Hilbert Transform and Lagrange interpolation, J. Approx. Theory, 60 (1990), 360-363. MR 1042655 (91a:42008)

[21] F. Peherstorfer, Weight functions admitting repeated positive Kronrod quadrature, BIT, 30 (1990), 145-151. MR.1032847 (91e:65043)

[22] F. Peherstorfer, On the Asymptotic Behaviour of Functions of the Second Kind and Stieltjes Polynomials and on the Gauss-Kronrod Quadrature Formulae, J. Approx. Theory, 70 (1992), 156-190. MR:1172017 (93h:42020)

[23] F. Peherstorfer, Stieltjes polynomials and functions of the second kind, J. Comput. Appl. Math., 65 (1995), 318-338. MR.1379141 (97c:33013)

[24] R. Piessens, E. de Doncker, C. Uberhuber and D. K. Kahaner, QUADPACK-A subroutine Package for Automatic Integration, Springer Series in Computational Mathematics 1, Berlin 1983. MR0712135 (85b:65022) 
[25] G. Szegő, Über gewisse orthogonale polynome, die zu einer oszillierenden Belegungsfunktion gehören, Math. Ann., 110 (1934), 501-513.

[26] G. Szegő, Orthogonal Polynomials, Amer. Math. Soc. Colloq. Publ., 23, American Mathematical Society, Providence, RI (1975). MR0372517 (51:8724)

[27] P. Vértesi, Hermite-Fejér interpolations of higher order. I, Acta Math. Hungar., 54(1-2) (1989), 135-152. MR1015784 (90k:41008)

Division of Applied Mathmatics, KAist, 373-1 Gusongdong, Yusongku, Taejon 305701, KOREA

E-mail address: hsjung@amath.kaist.ac.kr 